\title{
Dual-targeting nanoparticle vaccine elicits a therapeutic antibody response against chronic hepatitis B
}

\author{
Wenjun Wang ${ }^{1,2}$, Xiaoxiao Zhou ${ }^{1,2}$, Yingjie Bian ${ }^{1}$, Shan Wang ${ }^{3}$, Qian Chai', Zhenqian Guo ${ }^{3}$, \\ Zhenni Wang ${ }^{3}$, Ping Zhu ${ }^{4}$, Hua Peng ${ }^{1}{ }^{1}$, Xiyun Yan ${ }^{5}$, Wenhui Li ${ }^{6}$, Yang-Xin Fu ${ }^{7}$ and \\ Mingzhao Zhu (1) 1,2凶
}

Chronic hepatitis B is caused by prolonged infection with the hepatitis B virus (HBV), which can substantially increase the risk of developing liver disease. Despite the development of preventive vaccines against HBV, a therapeutic vaccine inducing an effective antibody response still remains elusive. The preS1 domain of the large HBV surface protein is the major viral attachment site on hepatocytes and thus offers a therapeutic target; however, its poor immunogenicity limits clinical translation. Here, we design a ferritin nanoparticle vaccine that can deliver preS1 to specific myeloid cells, including SIGNR1+ dendritic cells (which activate $\mathbf{T}$ follicular helper cells) and lymphatic sinus-associated SIGNR1 ${ }^{+}$macrophages (which can activate B cells). This nanoparticle vaccine induces a high-level and persistent anti-preS1 response that results in efficient viral clearance and partial serological conversion in a chronic HBV mouse model, offering a promising translatable vaccination strategy for the functional cure of chronic hepatitis $B$.

$\mathrm{H}$ epatitis $B$ is a major global health issue caused by the infection of hepatitis B virus (HBV), which attacks the liver and can cause both acute and chronic diseases ${ }^{1}$. It is estimated that 257 million people worldwide are HBV chronic carriers and have a high risk of developing liver disease, cirrhosis and hepatocellular carcinoma. Approximately 1 million chronic hepatitis $\mathrm{B}(\mathrm{CHB})$ patients die from these late complications each year. Conventional hepatitis B surface antigen (HBsAg) vaccination induces a protective antibody in most healthy vaccinated populations and has efficiently decreased the incidence rate of new HBV infections ${ }^{2}$. However, HBsAg fails to induce an effective antibody response in either preclinical animal models or patients with $\mathrm{CHB}$ infection, probably due to antigen-specific immune tolerance induced by the high load of viral antigens ${ }^{3}$. Therefore, an effective treatment strategy to eliminate and eradicate hepatitis B is highly desired.

Compared with HBsAg, the preS1 domain of the HBV large surface antigen has been suggested to be an ideal immunogen candidate for therapeutic vaccine development. PreS1 plays a pivotal role in HBV entry by interacting with sodium taurocholate cotransporting polypeptide (NTCP), identified as the cellular receptor for $\mathrm{HBV}^{4,5}$. Monoclonal antibodies against preS1 (MA18/7 and KR127) have been demonstrated to potently inhibit $\mathrm{HBV}$ infection in vivo and in vitro ${ }^{6,7}$. In addition, anti-preS1 can also eliminate $\mathrm{HBV}^{+}$cells via antibody-dependent cell-mediated cytotoxicity and phagocytosis ${ }^{8}$. Clinically, anti-preS1 is an early serum marker for HBV clearance ${ }^{9,10}$. All these data suggest that anti-preS1 response might be beneficial for $\mathrm{CHB}$ treatment. Moreover, in $\mathrm{CHB}$ patients, immune tolerance to preS1, if any, appears to be much lower than that to $\mathrm{HBsAg}^{3,11}$, as it only occurs in a trace amount compared with the latter in CHB patients ${ }^{12,13}$, which favours antibody response induction. In fact, we recently showed that preS1 antigen vaccination, but not HBsAg, induced a neutralizing therapeutic antibody response in HBV carrier mice ${ }^{11}$. Nonetheless, preS1 appears not to be an efficient antibody inducer compared with HBsAg. This is reflected under both natural infections and active vaccinations ${ }^{11,14,15}$. Therefore, how to improve the immunogenicity of preS1 for enhanced therapeutic antibody response is currently a key issue for preS1-based therapeutic vaccine development.

Nanoparticles (NPs) have emerged as an important platform for vaccine development. Lymph node (LN) antigen-presenting cell (APC) targeting has been frequently considered to be a valuable feature for many NP-based vaccines ${ }^{16}$. For preS1 vaccine, HBV core NPs have also been used ${ }^{7,17}$. However, given the complex composition of APCs and diversified functions of different APC subsets, the specific roles of different APC subsets during NP vaccine immunization seem to be vaguely understood ${ }^{18,19}$. Moreover, it has been unknown how to coordinately target them for an efficient adaptive immune response.

Ferritin self-assembling NPs have recently become widely appreciated for vaccine design ${ }^{20-24}$. However, the underlying immunological mechanism is still not fully understood. In the current study, we rationally designed a ferritin NP-preS1 vaccine. This vaccine demonstrates impressive preventive and therapeutic effects in mouse models. Mechanistically, the ferritin NPs are discovered to coordinately target distinct SIGNR1 ${ }^{+}$APCs for T follicular helper (Tfh) cell induction and B cell activation, leading to enhanced antibody production. Importantly, we have also uncovered a previously

'Key Laboratory of Infection and Immunity, Institute of Biophysics, Chinese Academy of Sciences, Beijing, China. ${ }^{2}$ College of Life Sciences, University of the Chinese Academy of Sciences, Beijing, China. ${ }^{3}$ Department of Pediatric Surgical Oncology, Children's Hospital, Chongqing Medical University, Chongqing, China. ${ }^{4}$ National Laboratory of Biomacromolecules, Institute of Biophysics, Chinese Academy of Sciences, Beijing, China. ${ }^{5}$ Key Laboratory of Protein and Peptide Pharmaceutical, Institute of Biophysics, Chinese Academy of Sciences, Beijing, China. ${ }^{6}$ National Institute of Biological Sciences, Beijing, China.

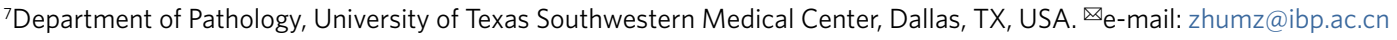


unrecognized mechanism: that macrophages capture NP-antigens for $\mathrm{B}$ cell activation in a CXCR5-dependent manner.

\section{Ferritin NP-preS1 induces efficient antibody response}

Ferritin NP-preS1 was generated taking advantage of a modified SpyTag/SpyCatcher technique ${ }^{25,26}$ (Fig. 1a). SpyTag-ferritin and $\Delta \mathrm{N}$ SpyCatcher-fused preS1 (SC-preS1) were both expressed in Escherichia coli. The self-assembly of SpyTag-ferritin NPs was suggested by size exclusion chromatography (Fig. 1b). SpyTag- and SpyCatcher-mediated covalent conjugation was confirmed by SDSpolyacrylamide gel electrophoresis (Fig. 1c). The ferritin NP-preS1 was further purified by size exclusion chromatography, and its slightly larger diameter was reflected by its earlier elution (Fig. 1d). The self-assembled NPs were confirmed using transmission electron microscopy (TEM) (Fig. 1e).

To assess the immunogenicity of the ferritin NP-preS1, naïve wildtype (WT) C57BL/6 mice were immunized as described in Methods. Ferritin NP-preS1 vaccine induced a 50 times higher antibody level than soluble SC-preS1 at day 21 (Fig. 1f). The anti-preS1 level continued to increase in the ferritin NP-preS1 immunization group and reached a peak around day 60, when an approximately 150 times higher antibody level was measured compared with the SC-preS1 control vaccine group, in which the antibody level still remained low. The antibody response gradually decreased but remained detectable at day 235. Furthermore, a boost immunization elicited a much stronger antibody response in the ferritin NP-preS1 group compared with the SC-preS1 control group (approximately 600-fold) (Fig. 1g). The enhanced immunogenicity was also seen in the absence of CpG-1826 adjuvant (Supplementary Fig. 1a), suggesting an intrinsic feature of ferritin NPs, probably due to repeated antigen display and an NP carrier effect ${ }^{16}$. However, in the presence of $\mathrm{CpG}$, the enhancement becomes more marked, suggesting a coordination between a ferritin NP intrinsic feature and an adjuvant-induced innate response.

The markedly enhanced antibody response is not a dose-dependent effect (Supplementary Fig. 1b). The SC portion itself that was fused to preS1 has no effect on the immunogenicity of SC-preS1 (Supplementary Fig. 1c). The foreignness of the bacterial ferritin is also not responsible for the enhanced antibody response, as preS1 displayed by a mouse ferritin $\mathrm{H}$ chain generated a comparable antibody level to bacterial ferritin (Supplementary Fig. 1d). The efficient antibody response is also not restricted to mouse strains (Supplementary Fig. 1e). In addition, physically mixing preS1 antigens with ferritin NPs instead of covalent binding could not elicit a strong anti-preS1 response, suggesting the necessity of antigen display on the ferritin NPs (Fig. 1h). Thus, preS1 conjugated to ferritin NPs generally enhanced the immunogenicity of preS1 antigen and elicited an efficient antibody response.

Further characterization showed that anti-preS1 antibody induced by ferritin NP-preS1 vaccination has higher avidity than that induced by SC-preS1 (Fig. 1i). IgG1, and not IgG2c, was the dominant anti-preS1 isotype after ferritin NP-preS1 immunization (Fig. 1j). Regarding the safety perspective, no cross-reactive antibodies to mouse ferritin were induced after Pyrococcus furiosus $(\mathrm{Pf})$ ferritin NP-preS1 immunization in mice, although anti- $P f$ ferritin was readily induced (Supplementary Fig. 1f). In addition, the serum levels of both glutamic-pyruvic transaminase and glutamic-oxaloacetic transaminase were normal (Supplementary Fig. 1g). The minimal iron content of ferritin NPs, compared with the iron storage capacity of 2,700 Fe per 24-mer for Pf ferritin ${ }^{27}$, was unlikely to affect iron homoeostasis in vivo (Supplementary Fig. 1h) ${ }^{20}$. Taken together, these results demonstrate notable immunogenicity and biosafety for the ferritin-NP-based preS1 vaccine.

\section{Protective and therapeutic effect of ferritin NP-preS1}

To test whether preS1 antisera induced by the ferritin NP-preS1 vaccine could prevent $\mathrm{HBV}$ infection, vaccine-immunized mice were challenged with adeno-associated virus (AAV)-HBV1.3 two weeks after the second immunization (Fig. 2a). PreS1 antigen and HBV DNA in the sera were greatly inhibited in the ferritin NPpreS1 vaccine group compared with the control groups (Fig. 2b,c), indicating effective clearance of infectious HBV particles in vivo, probably due to rapid elimination.

To evaluate the neutralizing capability of the antisera, HepG2hNTCP cells were infected with HBV in the presence of sera from different immunization groups as described previously ${ }^{28}$. The levels of $\mathrm{HBsAg}$ and $\mathrm{HBeAg}$ in cell culture media were significantly inhibited by the sera from ferritin NP-preS1-immunized mice, in stark contrast with the preS1-immunization group (Fig. 2d,e). To more accurately measure $\mathrm{HBV}$ infection inhibition, the levels of $\mathrm{HBV}$ 3.5-kb RNA and HBV total RNA, indicating HBV viral replication in HepG2-hNTCP cells, were also determined, and significant inhibition by the sera of the ferritin NP-preS1 group but not the control groups was found (Fig. 2f). These results confirm that the antisera to preS1 elicited by the ferritin NP-preS1 vaccine can effectively prevent HBV infection of hepatocytes in vitro. Thus, both in vivo and in vitro results suggest that the ferritin NP-preS1 vaccine is an effective $\mathrm{HBV}$ prophylactic vaccine.

To determine the therapeutic effect of the ferritin NP-preS1 vaccine, $\mathrm{HBV}$ carrier mice were established ${ }^{11}$, and immunized with equimolar ferritin NP-preS1 vaccine or SC-preS1 four times every two weeks (Fig. 3a). There is no detectable anti-preS1 response in the established HBV carrier mice, consistent with the clinical observa$\operatorname{tion}^{29,30}$. However, after the second boost on day 63, the ferritin NPpreS1 vaccine induced a significant amount of anti-preS1, which was barely detectable in the SC-preS1 group (Fig. 3b). Meanwhile, preS1 antigen and HBsAg in the sera declined significantly with the induction of anti-preS1 (Fig. 3c,d). On day 147, about 10 weeks after the last immunization, serum HBV DNA was also significantly reduced in the ferritin NP-preS1 group but not in the control groups (Fig. 3e). Moreover, in four out of seven mice in the ferritin NP-preS1 group, serum HBV DNA dropped to undetectable levels, also showing barely detectable HBsAg and significant amounts of anti-HBs (Fig. 3e), suggesting potential for a functional cure. In addition, the HBV core antigen as detected by immunological histological chemistry staining (Fig. 3f) and covalently closed circular DNA (cccDNA) as detected by quantitative real-time PCR (Fig. 3g) were also significantly diminished in the liver of the ferritin NPpreS1 group. The clearance of HBV core antigen in the liver was not accompanied by liver injury (Fig. 3h). Thus, ferritin-preS1 NPs plus CpG functioned as an effective and non-pathogenic therapeutic vaccine in the mouse model of protracted HBV infection.

Interferon- $\gamma$ (IFN- $\gamma$ ) is an important activator for antibodydependent cell-mediated cytotoxicity and phagocytosis ${ }^{31}$, which have been suggested to mediate anti-preS1 for therapeutic effect ${ }^{8}$. On ferritin NP-preS1 vaccination, significant numbers of preS1specific IFN- $\gamma$-producing $\mathrm{T}$ cells were detected using enzymelinked immune absorbent spot (ELISpot) assay in LNs, spleens and liver tissues (Supplementary Fig. 2a). Furthermore, IFN- $\gamma$ blockade with a blocking antibody (XMG1.2) almost completely abolished the therapeutic effect on HBV core antigen clearance (Supplementary Fig. 2b,c). These data suggest that the Th1 response induced by ferritin NP-preS1 vaccine might play an important role in achieving therapeutic efficacy: enhancing IFN- $\gamma$ response may synergize with ferritin NP-preS1 vaccine.

\section{Ferritin NP vaccine targets distinct APCs}

To understand how the ferritin NP vaccine is recognized and processed by the host immune system, fluorescein-labelled ferritin NPs were generated to trace their path. On subcutaneous injection, ferritin NP-eGFP (enhanced green fluorescent protein) was abundantly located in the medullary regions and also but to a lesser extent in the interfollicular region (Fig. 4a). Interestingly, ferritin NP-eGFP 


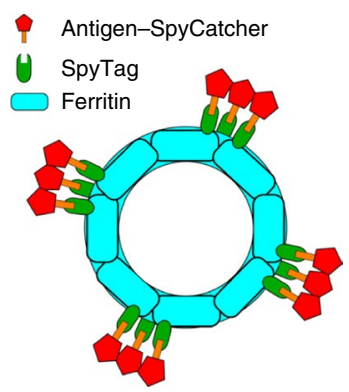

d
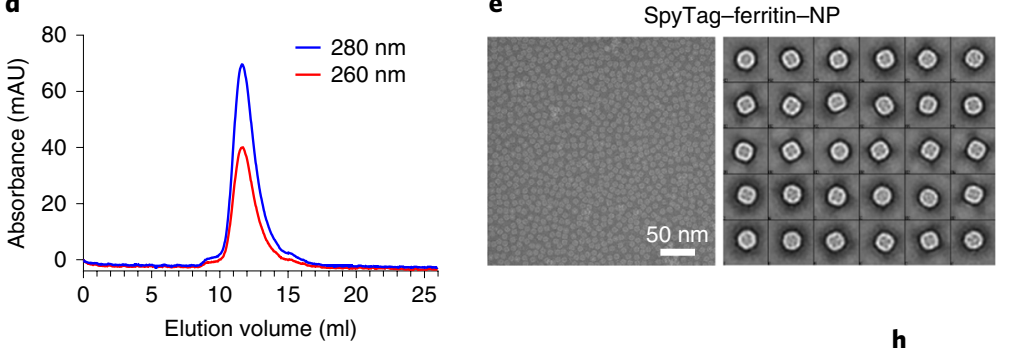

e

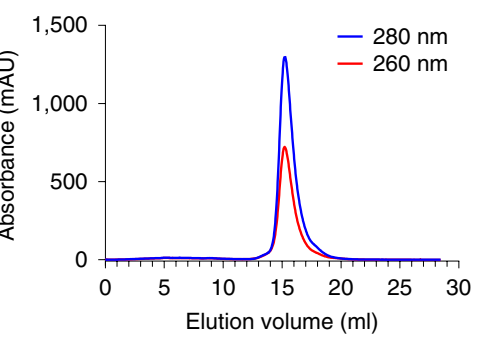

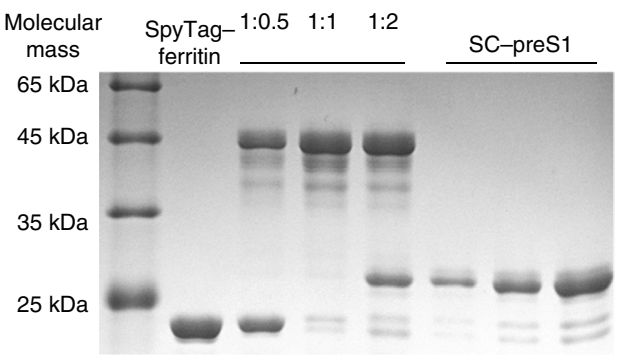

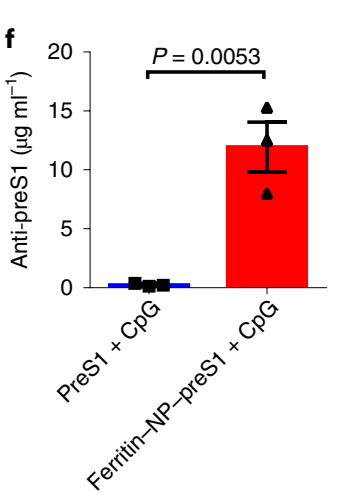

i

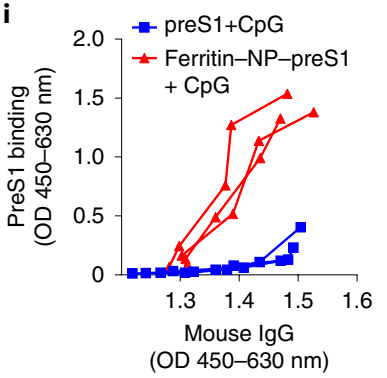

h

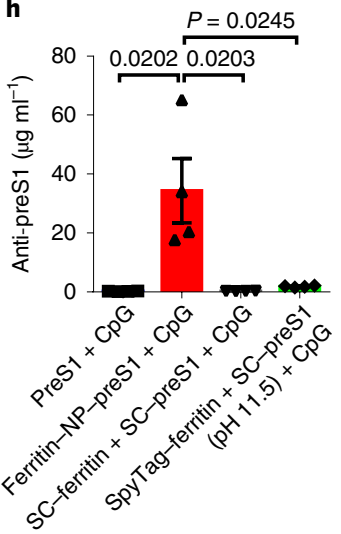

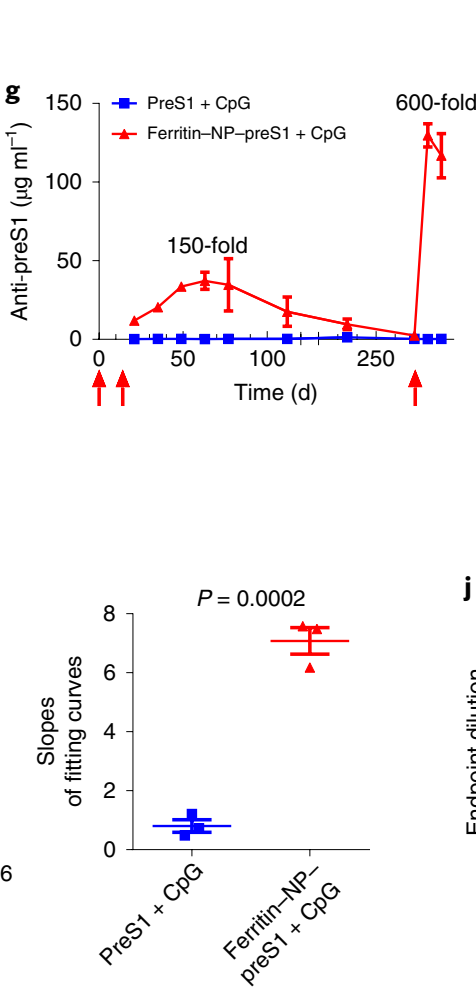

Ferritin-NP-pres1

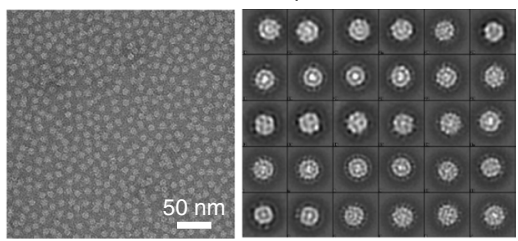

j

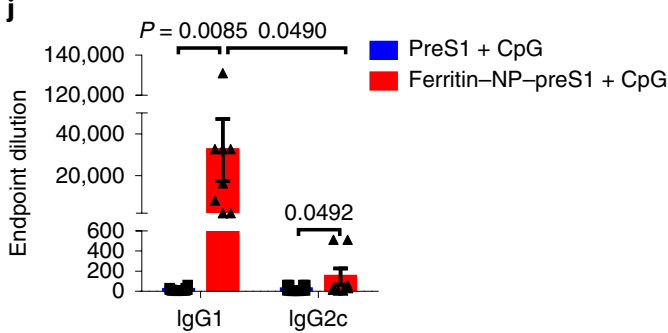

Fig. 1 | Molecular design and characterization of ferritin NP vaccine. a, Schematic illustration of ferritin-based vaccine delivery vehicle. $\mathbf{b}$, Size exclusion chromatography of SpyTag-ferritin NPs. The ultraviolet absorptions at $280 \mathrm{~nm}$ and $260 \mathrm{~nm}$ are shown. AU, absorbance unit. c, SDS-polyacrylamide gel electrophoresis analysis of the covalent ligation of SpyTag-ferritin $(22.4 \mathrm{kDa})$ and SC-preS1 $(\sim 25.4 \mathrm{kDa})$ at 1:0.5, 1:1 and 1:2 mole ratios at $4{ }^{\circ} \mathrm{C}$ overnight. d, Size exclusion chromatography of ferritin NP-preS1. e, TEM images and two-dimensional reconstruction of SpyTag-ferritin NP and ferritin NP-preS1. Panels b-e show representative results of at least three independent experiments. f, Anti-preS1 response at vaccine immunization on day 21 was analysed using enzyme-linked immunosorbent assay (ELISA) ( $n=3$ mice per group). Data are representative of five independent experiments. $\mathbf{g}$, Kinetics of antipreS1 levels after immunization $(n=3)$. The red arrows indicate the immunization times. $\mathbf{h}$, Comparison of conjugated and unconjugated vaccines on anti-preS1 response. i, Anti-preS1 IgG avidity on vaccine immunization was detected with ELISA. Each curve represents an individual serum sample ( $n=3$ ) (left). The slopes of each fitting curve were calculated (right). OD, optical density. $\mathbf{j}$, Titres of preS1-specific IgG1 and IgG2c in the sera from ferritin NP-preS1 vaccine $(n=8)$ or SC-preS1 $(n=14)$ immunized mice on day $21 . \mathbf{g}-\mathbf{j}$ show representative results of three independent experiments. In $\mathbf{f}-\mathbf{j}$, data are shown as mean \pm s.e.m.; statistical significance was determined using an unpaired two-tailed $t$-test. .

was highly colocalized with cells with SIGNR1 expression. SIGNR1 mediates the recognition of several virus particles ${ }^{32,33}$. This finding prompted us to hypothesize that ferritin NP-eGFP was actively captured by SIGNR $1^{+}$cells during their passive transportation and retained in LNs. To further confirm this and examine the ferritin NP cell-targeting property in more detail, ferritin NP capture by 
a

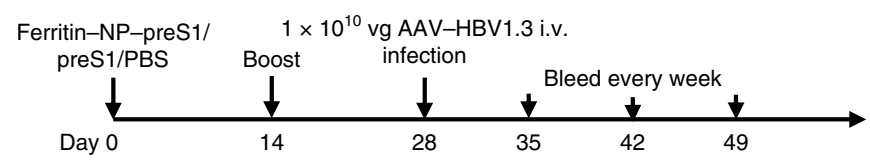

b
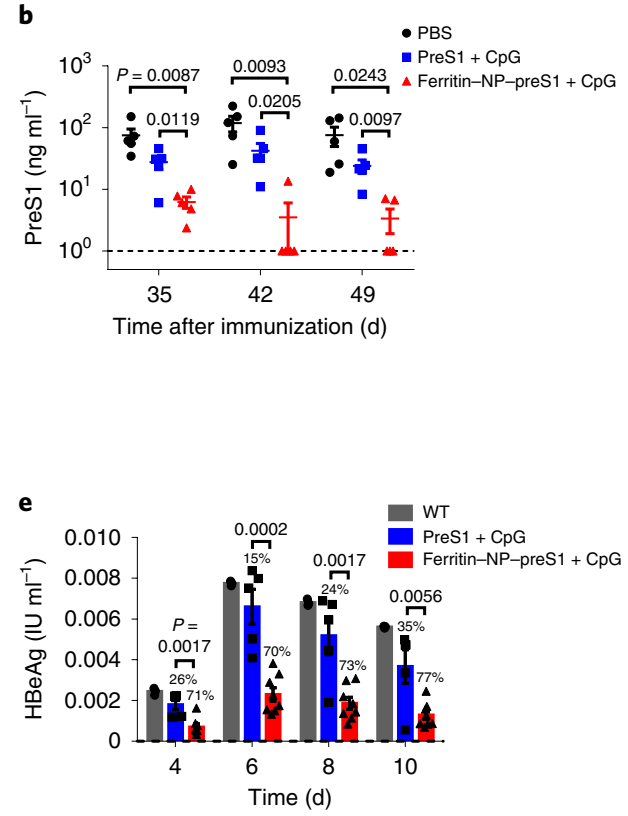

c
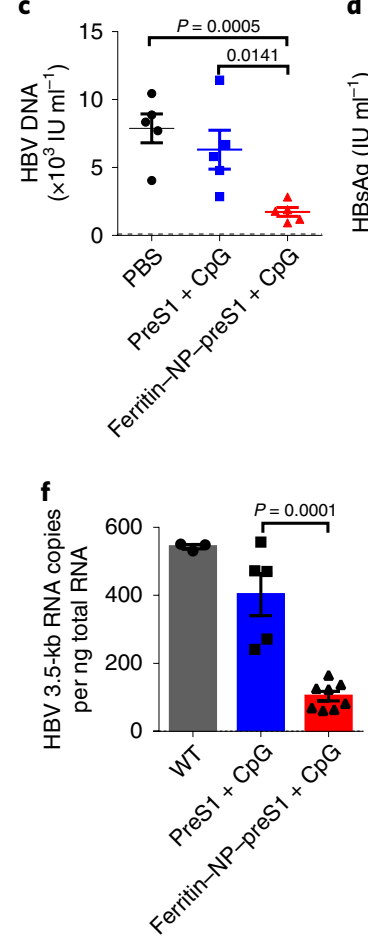
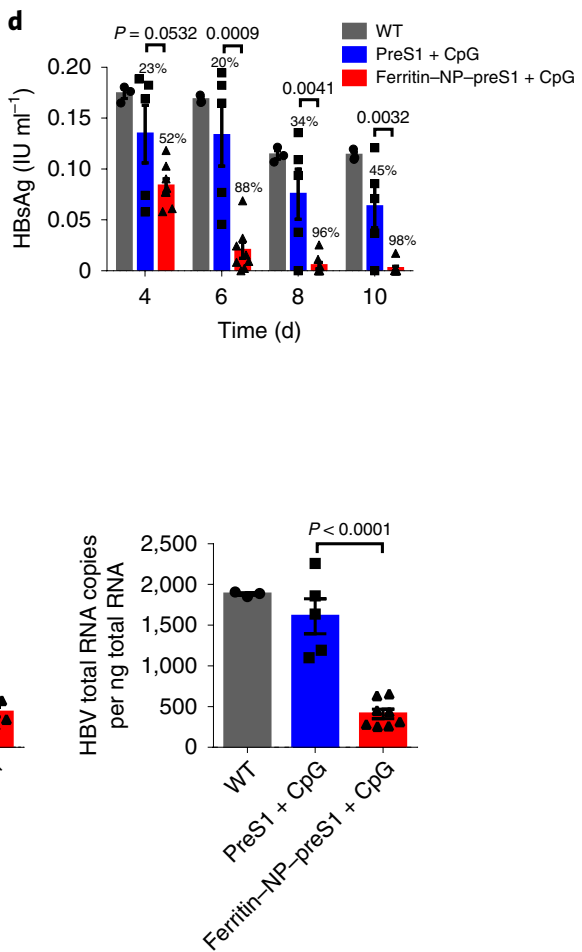

Fig. 2 | Protective immunity of ferritin NP-preS1 vaccine against HBV. a, Naïve WT C57BL/6 mice $(n=5)$ were subcutaneously immunized with 200 pmol ferritin NP-preS1 vaccine or equimolar SC-preS1 soluble antigen with $30 \mu \mathrm{g}$ CpG-1826 on days 0 and 14 . On day 28 , mice were infected with $1 \times 10^{10}$ viral genome equivalents (vg) AAV-HBV1.3 virus intravenously (i.v.). Blood samples were collected every week from day 35. b. The serum level of preS1 after AAV-HBV1.3 infection was detected using ELISA. c, The serum HBV DNA level on day 35 was quantified by real-time PCR. $\mathbf{b}$ and $\mathbf{c}$ show representative results of three independent experiments. d-f, HepG2-hNTCP cells were incubated with $1 \times 10^{7} \mathrm{vg} \mathrm{HBV}$ in the presence of immune sera collected from ferritin NP-preS1 $(n=8)$ or SC-preS1 $(n=5)$ immunized mice or WT mice $(n=3)$. After $24 \mathrm{~h}$ incubation, HepG2-hNTCP cells were washed and cultured in maintenance medium for $10 \mathrm{~d}$. The culture supernatant was changed and collected every $2 \mathrm{~d}$. d,e, Levels of $\mathrm{HBs} \mathrm{Ag}$ (d) and $\mathrm{HBeAg}$ (e) in the supernatant were measured using ELISA. On day 10, HepG2-hNTCP cells were collected in TRIzol and RNA was extracted. f, HBV 3.5-kb RNA and HBV total RNA were quantified using real-time PCR. The detection limits in $\mathbf{b}-\mathbf{f}$ are shown as dashed lines. $\mathbf{d}-\mathbf{f}$ show representative results of two independent experiments. In b-f, data are shown as mean \pm s.e.m.; statistical significance was determined using an unpaired two-tailed $t$-test.

phagocytic cells was determined by flow cytometry. Supportively, SIGNR1 $^{+}$resident dendritic cells (rDCs) and migratory DCs (miDCs) (Supplementary Fig. 3a) showed significantly higher GFP immunofluorescence intensity than their SIGNR1- counterparts, indicating the preferential capture of ferritin NPs by SIGNR1 $1^{+}$ DCs (Fig. 4b). Similarly, in non-DC cells, the SIGNR $1^{+}$population, which was mostly $\mathrm{CD} 169^{+}$macrophages, also showed preferential capture of ferritin NPs (Fig. 4c). As control, the soluble eGFP was almost undetectable in either SIGNR1 $1^{+}$or SIGNR1- subsets. Similar results were found when fluorescein isothiocyanate (FITC) was used to label ferritin NP-preS1 or SC-preS1 (Supplementary Fig. 3b-d). The CpG-1826 adjuvant had no influence on these captures (Supplementary Fig. 4). Therefore, ferritin NPs appear to be predominantly captured by both SIGNR $1^{+}$DCs and SIGNR $1^{+}$ macrophages in the draining LNs (dLNs).

To further evaluate the role of SIGNR1 ${ }^{+}$cells in ferritin NPeGFP capture, clodronate liposome (CLL) was injected into the footpads (f.p.) to eliminate APCs from popliteal LNs (pLNs) ${ }^{34}$. Both SIGNR1 ${ }^{+}$DCs and SIGNR1 ${ }^{+}$macrophages were efficiently depleted (Supplementary Fig. 3e). The capture of ferritin NP-eGFP was almost completely lost after CLL treatment (Fig. 4d,e), suggesting an essential role of SIGNR1 ${ }^{+}$cells in ferritin NP retention.
This preferential capture by SIGNR1+ APCs is not only because of their location-determined accessibility to injected ferritin NPs. In fact, in vitro coculture of digested single-cell suspensions from LNs with ferritin NPs demonstrated similar preferential capture by SIGNR1 ${ }^{+}$APCs (Supplementary Fig. 5), suggesting an intrinsic feature of ferritin NPs or SIGNR $1^{+}$APCs.

Although SIGNR1 expression is highly positively correlated with ferritin NP capture, it does not seem to be essential, at least on its own, for ferritin NP capture, as shown in the data obtained from Signr1-deficient mice (Supplementary Fig. 6). Given the presence of several other orthologues of SIGNR1 in mouse ${ }^{35,36}$, the exact function of SIGNR1 and this gene family in ferritin NP capture remains to be determined in the future.

To further determine whether this preferential targeting is transferable to the human situation, we have tested its capture using digested single cells from human LNs. The human homologue of murine SIGNR1 is DC-SIGN (CD209), not only in terms of genomic structure, but also for cell distribution and function in $\mathrm{LNs}^{37,38}$. Flow cytometric analysis showed that ferritin NPs were indeed preferentially captured by $\mathrm{DC}-\mathrm{SIGN}^{+}$macrophages compared with DC-SIGN ${ }^{-}$macrophages (Supplementary Fig. 7a), probably supporting the feasibility of clinical translation. 
a
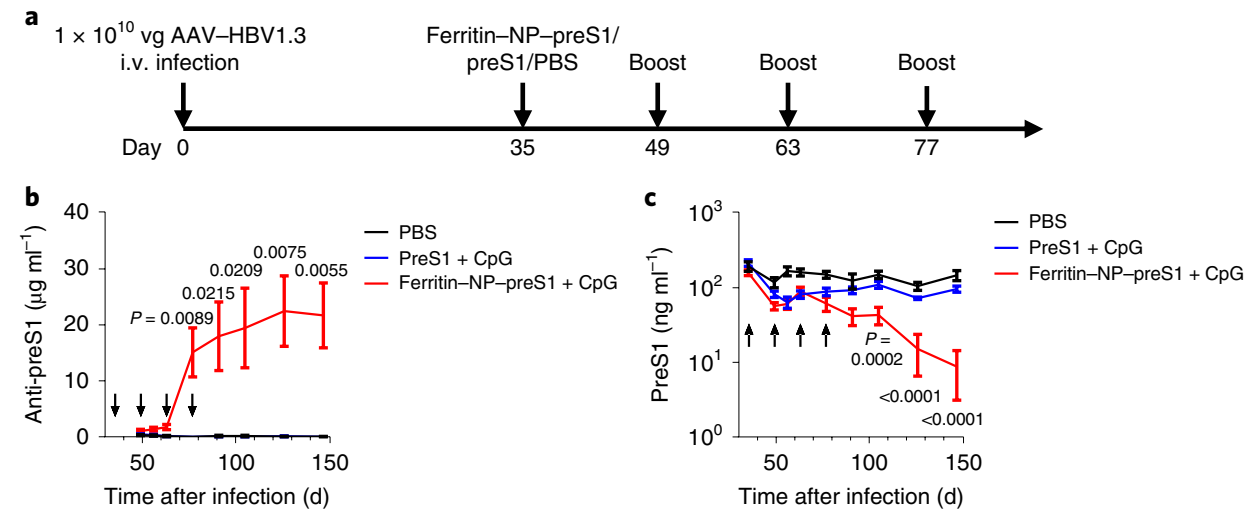

d
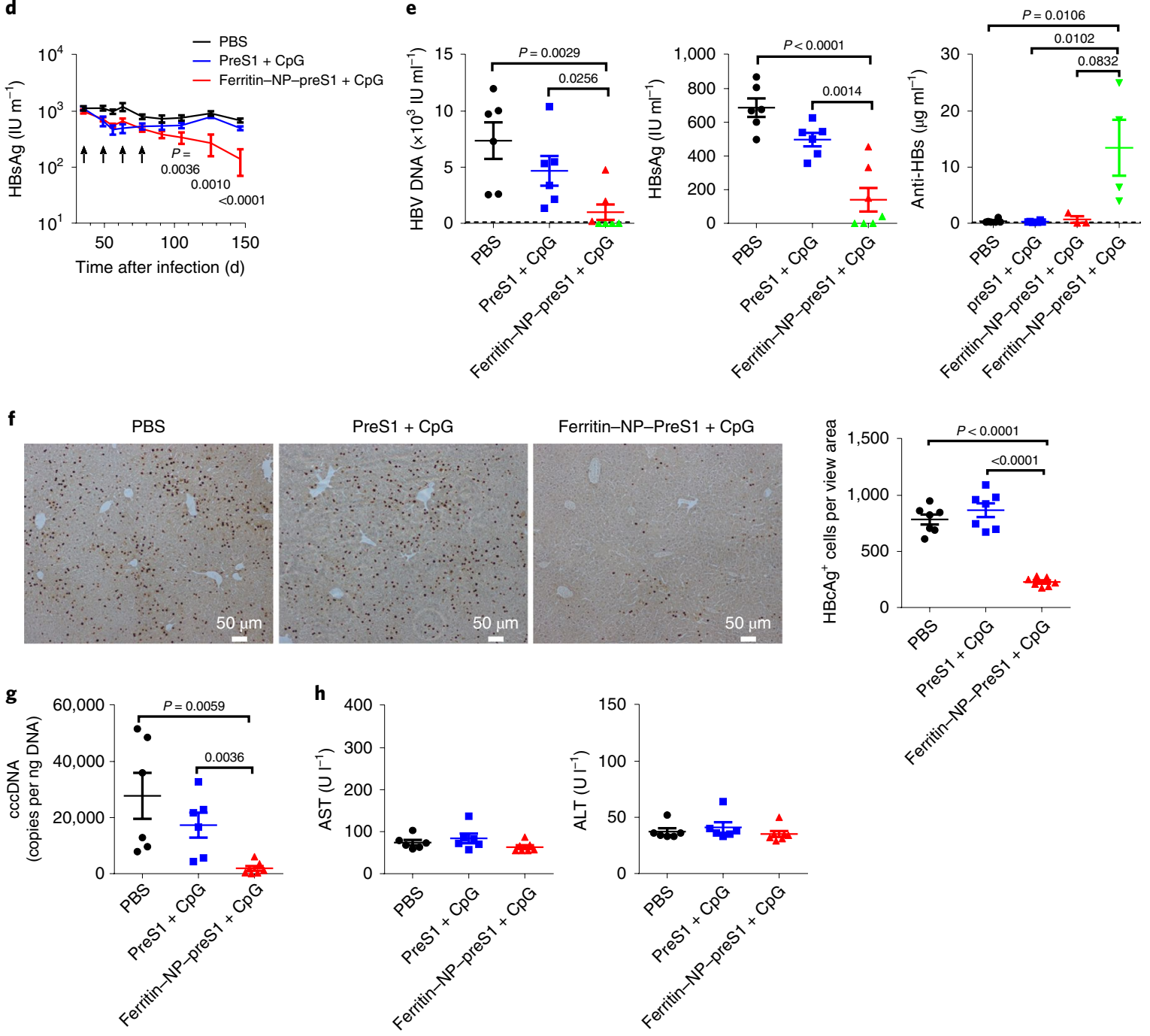

Fig. 3 | Ferritin NP-preS1 as therapeutic vaccine. a, C57BL/6 mice were inoculated with $1 \times 10^{10} \mathrm{vg} \mathrm{AAV-HBV1.3} \mathrm{virus} \mathrm{intravenously.} \mathrm{After} 5$ weeks, stable HBV carrier mice were vaccinated with 500 pmol ferritin NP-preS1 vaccine $(n=7)$ or equimolar SC-preS1 soluble antigen $(n=6)$ with $30 \mu g$ CpG-1826 adjuvant four times every 2 weeks. Sera were collected every 2 weeks. b-d, Serum levels of anti-preS1 (b), preS1 antigen (c) and HBsAg (d) were determined using ELISA. There is no difference between PBS $(n=6)$ and SC-preS1, and the $P$ values shown in the figure are statistical significances between PBS and ferritin NP-preS1. IU, international units. e, Serum levels of HBV DNA, HBsAg and anti-HBs on day 147. Green dots represent the same mice across the three panels with undetectable HBV DNA. f, Immunological histological chemistry staining for hepatitis B core antigen (HBcAg) in hepatocytes on day 147. Positive cells were counted using Image J software in each section field ( $n=7$ from three mice). $\mathbf{g}$, HBV cccDNA in the liver quantified using real-time PCR on day 147. h, Glutamic-pyruvic transaminase (ALT) and glutamic-oxaloacetic transaminase (AST) measured for the sera collected on day 147. b-h show representative results of four independent experiments. Data are shown as mean \pm s.e.m.; statistical significance was determined using an unpaired two-tailed $t$-test. 

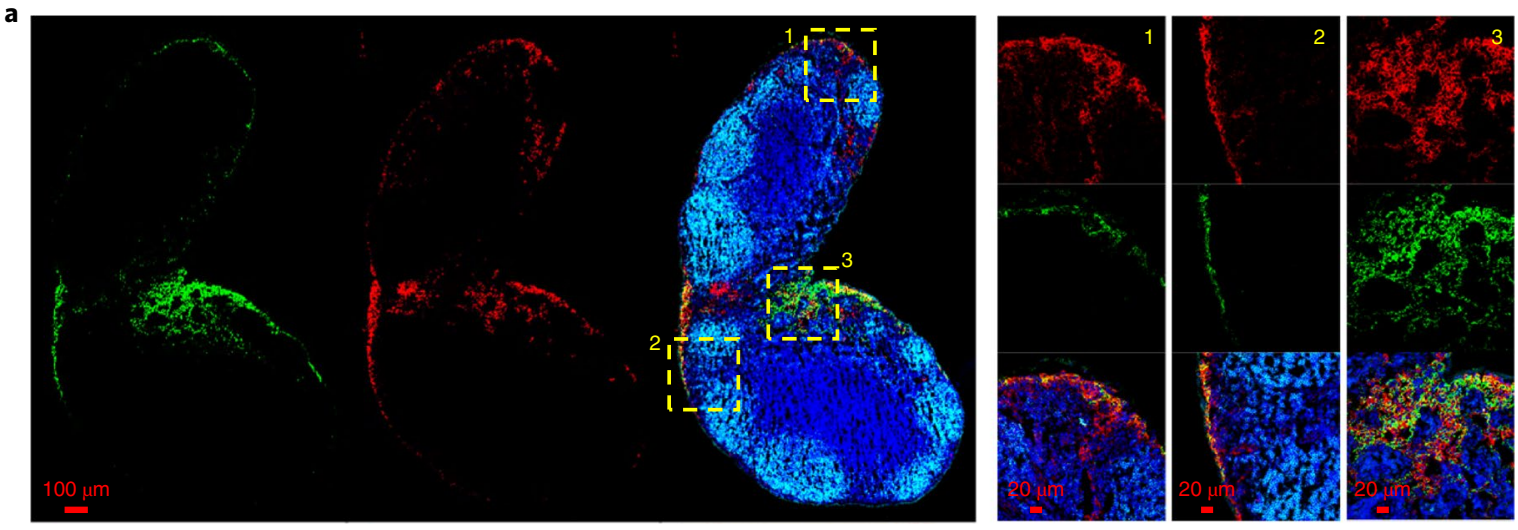

b

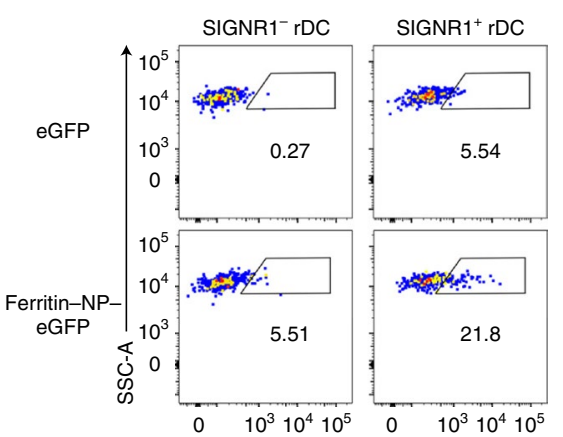

eGFP/ferritin-NP-eGFP

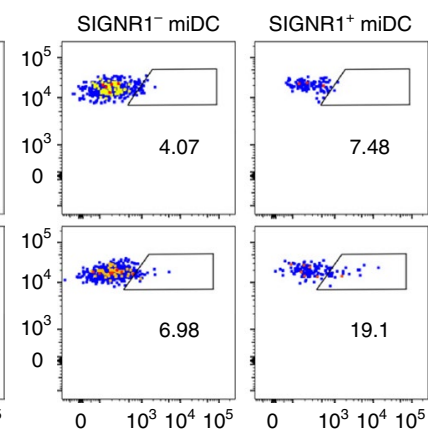

Ferritin-NP_eGFP SIGNR1 B220 DAPI

c

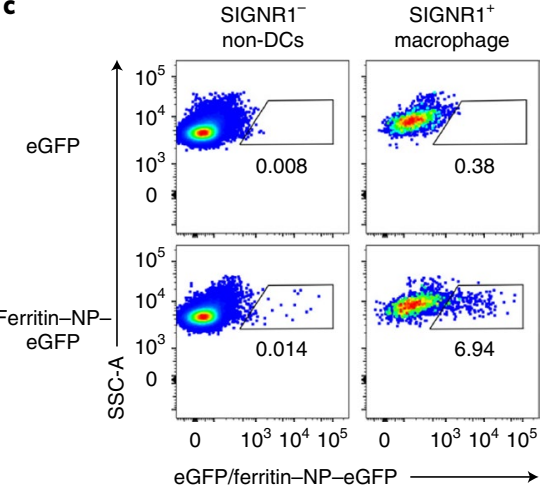

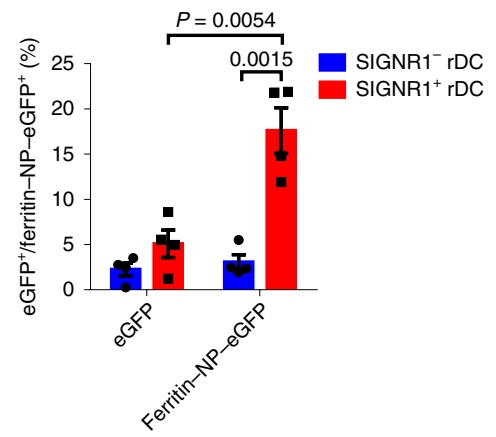
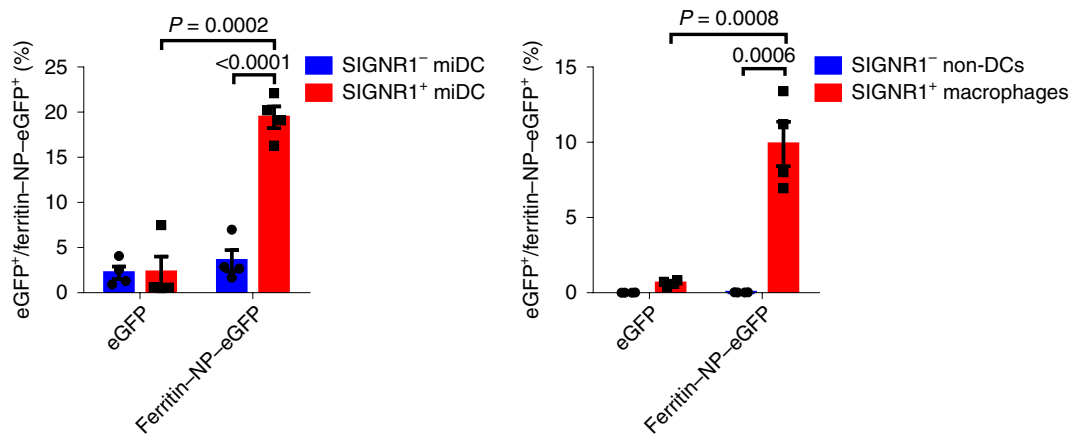

d

CON

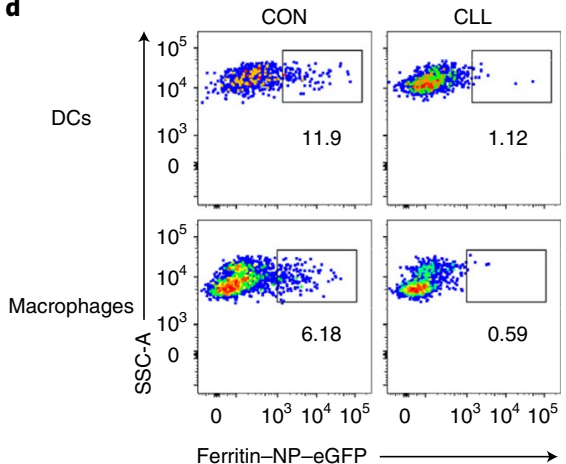

CON

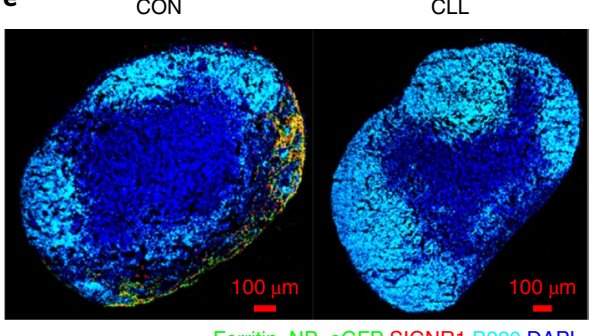

Fig. 4 | Ferritin NP vaccine targets distinct APCs. a, C57BL/6 mice were subcutaneously injected with 2 nmol ferritin NP-eGFP. $4 \mathrm{~h}$ after injection, cryosections of inguinal LNs (iLNs) were obtained. The section was stained with anti-GFP, anti-SIGNR1, anti-B220 and 4,6-diamidino-2-phenylindole (DAPI). Three representative areas of interfollicular region and medulla are shown in magnification. Representative results of four independent experiments. $\mathbf{b}, \mathbf{c}$, $\mathrm{C} 57 \mathrm{BL} / 6$ mice were subcutaneously injected with 2 nmol ferritin NP-eGFP $(n=4)$ or equimolar eGFP-SpyTag $(n=4)$. $4 \mathrm{~h}$ after injection, iLNs were digested into single cells and the capture of ferritin NP-eGFP or eGFP-SpyTag by the indicated DCs (b) or non-DCs (c) was presented and statistically analysed. Numbers adjacent to the outlined areas indicate the percentage of each gate. Representative results of three independent experiments. Data are shown as mean \pm s.e.m.; statistical significance was determined using an unpaired two-tailed t-test. SSC-A, side scatter area. d, 10 d after CLL or control liposome (CON) f.p. injection, the capture of ferritin NP-eGFP by DCs or CD11c-CD11b+ macrophages was analysed. e, $10 \mathrm{~d}$ after CLL or CON treatment, ferritin NP-eGFP accumulation in pLNs $2 \mathrm{~h}$ after f,p. injection was determined by immunofluorescence microscopy. $\mathbf{d}$ and e show representative results of three independent experiments. 


\section{Coordination of SIGNR1+ DCs and macrophages}

Germinal centre (GC) formation is essential for a strong antibody response, and the affinity selection of GC B cells is dependent on $\mathrm{CD}^{+}{ }^{+}$Tfh cells $^{39}$. Employing the well established OVA-OT-II antigen-specific $\mathrm{CD}^{+} \mathrm{T}$ cell system, we found that ferritin NP-OT-II induced about eight times as many $\mathrm{CXCR}^{+} \mathrm{PD} 1^{+}$and $\mathrm{PD} 1^{+} \mathrm{GL} 7^{+}$ Tfh cells compared with control SC-OT-II $6 \mathrm{~d}$ after immunization (Fig. 5a). Furthermore, about 11 times more GC B $\left(\mathrm{FAS}^{+} \mathrm{GL}^{+}\right)$cells were detected in the ferritin NP-preS1 vaccine group compared with the SC-preS1 control group (Fig. 5b). Histological analysis revealed that GC numbers in ferritin NP-preS1-immunized dLNs were also significantly higher than those in the SC-preS1 group (Fig. 5c). Therefore, ferritin NP vaccine is an efficient antigen-delivery platform to enhance Tfh and GC B responses.

All rDCs, miDCs and SIGNR1 ${ }^{+}\left(\mathrm{CD} 169^{+}\right)$macrophages efficiently capture ferritin NPs on immunization. To study which cells induce $\mathrm{T}$ cell activation, dLNs were collected $12 \mathrm{~h}$ after ferritin NP-OT-II vaccine immunization. Four populations of APCs were sorted according to the gating strategy (Supplementary Fig. 8) and cocultured with OT-II T cells. Macrophages did not induce a detectable level of OT-II T cell activation or proliferation (Fig. 5d,e). Interestingly, rDCs induced a significantly higher degree of $\mathrm{T}$ cell activation and proliferation than miDCs (Fig. 5d,e). Therefore, rDCs appear to play a major role in Tfh generation compared with miDCs.

For particulate antigen immunization, DCs can carry antigens to interfollicles for $\mathrm{B}$ cell activation ${ }^{32}$. To directly determine the role of DCs in B cell activation in the current scenario, rDCs and miDCs were depleted in CD11c-DTR (diphtheria toxin receptor) bone marrow chimaeric mice with diphtheria toxin (DT), with PBS-treated mice as controls (Fig. 5f). DC depletion resulted in a lower titre of IgG response, indicating that DCs played an essential role in T-cell-dependent antibody response (Fig. 5g). However, IgM antibodies, produced by early-activated B cells largely in a T-cellindependent manner ${ }^{32}$, were not affected (Fig. $5 \mathrm{~g}$ ). Thus, while DCs play a major role in $\mathrm{T}$ cell activation, they are dispensable for early $B$ cell activation during ferritin NP vaccine immunization.

In addition to DCs, SIGNR1 ${ }^{+}$macrophages captured ferritin NP efficiently (Fig. 4c). SIGNR $1^{+}$macrophages showed markedly reduced transcript abundance for lysosome-associated membrane proteins-1/2, lysozyme and lysosomal proteases compared with SIGNR1 ${ }^{-} \mathrm{F} 4 / 80^{+}$(abbreviated as $\mathrm{F} 4 / 80^{+}$) macrophages (Supplementary Fig. 9a), indicating their poor degradation ability and potential role as antigen retainers for B cell activation. To directly test this, four subsets of APCs (miDCs, rDCs, SIGNR1+ macrophages and $\mathrm{F} 4 / 80^{+}$macrophages) were isolated as shown in Supplementary Fig. 8 from dLNs $3 \mathrm{~d}$ after ferritin NP-HEL (hen egg lysozyme) immunization and were incubated with MD4 $\mathrm{B}$ cells in vitro for $24 \mathrm{~h}$. Except for SIGNR1 ${ }^{+}$macrophages, no other populations activated MD4 B cells as demonstrated by the upregulation of CD69 and CD86 (Fig. 6a), indicating a durable retention of antigen by SIGNR1 ${ }^{+}$macrophages. In vivo, a CSF1R-blocking antibody-mediated SIGNR1 ${ }^{+}$macrophage depletion (Supplementary Fig. $9 \mathrm{~b})^{33}$ resulted in significantly reduced anti-preS1 $\operatorname{IgM}$ and IgG $10 \mathrm{~d}$ after the first immunization (Fig. 6b). On day 21, significantly lower levels of anti-preS1 IgG and fewer preS1-specific IgG antibody-secreting cells (ASCs) were also observed in anti-CSF1Rtreated mice (Fig. 6c,d). Thus, SIGNR1 ${ }^{+}$macrophages play a vital role in early B cell activation and IgG response.

SIGNR $1^{+}$macrophages are mainly distributed in medulla and interfollicular regions in LNs. We wondered how they can transfer antigen for B cell activation. On CpG immunization, a cluster of SIGNR1 ${ }^{+}$cells emerged in the follicular regions of dLNs (Fig. 6e, top panel), which express CD169 rather than CD11c, indicating their macrophage identity (Supplementary Fig. 10a). This suggested that SIGNR1 ${ }^{+}$macrophages might directly carry antigens to follicles for B cell activation. To understand how this migration is regulated, we examined the CXCL13-CXCR5 axis, given the abundant expression of CXCL13 in B cell follicles and its important role for follicular migration ${ }^{40}$. In $C x c r 5^{-/-}$mice, no SIGNR1 $1^{+}$cells could be found in $\mathrm{B}$ cell follicles on immunization, in stark contrast to WT mice (Fig. 6e, bottom panel). In supporting these data, flow cytometric analysis showed that SIGNR1 ${ }^{+}$macrophages, but not SIGNR1 ${ }^{-}$macrophages or SIGNR1+ DCs, significantly upregulated CXCR5 on immunization (Supplementary Fig. 10b). The upregulation of CXCR5 appears to be a direct effect of $\mathrm{CpG}$ and unique to macrophages, as CpG alone induces CXCR5 expression on isolated primary SIGNR $1^{+}$macrophages but not DCs under in vitro culture (Supplementary Fig. 10c). Given the aberrant LN organization in Cxcr5 $5^{-/}$mice, WT:Cxcr5 ${ }^{-/-}$mixed bone marrow chimaeric mice were generated and confirmed the macrophage cell intrinsic role of CXCR5 on their migration to B cell follicles (Fig. 6f).

To further evaluate the role of CXCR5 and follicular migration of SIGNR $1^{+}$macrophages in B cell activation, we injected ferritin NP-HEL-carrying WT or Cxcr5 $5^{-1-}$ SIGNR1 ${ }^{+}$macrophages into the f.p. of naive CD45.1 mice, who had received MD4 B cells (CD45.2) the day before. $24 \mathrm{~h}$ later, MD4 B cell activation was determined by flow cytometry. The result showed that, while ferritin NP-HELcarrying WT SIGNR1+ macrophages activated MD4 B cells significantly, Cxcr5 ${ }^{-1-}$ SIGNR1 $1^{+}$macrophages were unable to do so (Fig. $6 \mathrm{~g}$ ). Thus, these data together suggest a previously unrecognized mechanism for B cell activation via CXCR5-dependent SIGNR1 ${ }^{+}$macrophage follicular migration and antigen transportation.

\section{Conclusions}

CHB therapy has different levels of goals: complete sterilizing cure, functional cure and partial cure ${ }^{41,42}$. So far, although complete cccDNA elimination still appears a great challenge, functional cure, which is defined as sustained, undetectable serum HBsAg and HBV DNA with or without seroconversion to anti-HBs after a finite course of therapy, but with the persistence of residual cccDNA, is more realistic. In our study, we have found significantly reduced serum HBsAg and HBV DNA after four vaccinations of ferritin NP-preS1. Furthermore, some mice in the ferritin NP-preS1 group

Fig. 5 | Ferritin NP vaccine induces efficient Tfh and GC responses. a, Naïve WT Thy 1.2 C57BL/6 mice $(n=3)$ were adoptively transferred with $1 \times 10^{7}$ Thy $1.1^{+}$OT-II splenocytes and immunized. On day 6, CD4+Thy1.1+TCR V $\alpha 2^{+}$OT-II Tfh cells from LNs were analysed by flow cytometry (TCR, T-cell antigen receptor). The percentage and quantification of OT-II Tfh cells are shown. $\mathbf{b}$, On day 63 of immunization (peak anti-preS1 response), GC B cells from iLNs were analysed by flow cytometry. $\mathbf{a}$ and $\mathbf{b}$ show representative results of three independent experiments. $\mathbf{c}$, Cryosections of iLNs were stained with anti-B220, anti-GL7 and DAPI. The number of GCs was counted. Data were pooled from $n=30$ slide sections from 10 mice in three independent experiments. d,e, $12 \mathrm{~h}$ after immunization, rDCs (CD103-), miDCs $\left(C D 103^{+}\right), \mathrm{CD}^{-} 69^{+}$macrophages $\left(\mathrm{CD} 11 \mathrm{c}^{-} \mathrm{CD} 11 \mathrm{~b}^{+} \mathrm{CD} 169^{+}\right)$and F4/80+ macrophages $\left(C D 11 c^{-} C D 11 b^{+} C D 169-F 4 / 80^{+}\right)$were sorted from dLNs, and then incubated with naïve OT-II T cells $(n=3$ culture wells). OT-II T cell activation (d) and carboxyfluorescein succinimidyl ester (CFSE)-labelled OT-II T cell proliferation (e) were determined by flow cytometry at $24 \mathrm{~h}$ and $72 \mathrm{~h}$, respectively. Representative results of five independent experiments. iono, ionomycin. f, Flow cytometry analysis confirmed DC depletion in iLNs of CD11c-DTR bone marrow chimaeric mice treated with DT. Data show representative results of three independent experiments. $\mathbf{g}$, PreS1-specific IgM and IgG responses were detected in CD11c-depleted mice on day 10 at immunization $(n=4)$. Data are representative of three independent experiments. In a-e and $\mathbf{g}$, data are shown as mean \pm s.e.m.; statistical significance was determined using an unpaired two-tailed $t$-test. 
showed undetectable HBsAg and HBV DNA in the sera, and more impressively these mice also showed significant amounts of antiHBs. These data indicate that ferritin NP-preS1 vaccine may be a good candidate to achieve a functional cure. Moreover, encouragingly, ferritin NP-preS1 vaccine also significantly reduced HBV cccDNA in the liver. Thus, our study might provide a new strategy toward eliminating cccDNA that could be worthy of future evaluation when used either alone or in combination ${ }^{43}$.

Ferritin is a unique NP vector that recently drew a lot of attention and showed an impressive antibody-enhancing effect for several other important viral antigens $\mathrm{s}^{20-24}$. However, the underlying immunological mechanism is still poorly understood, which might limit
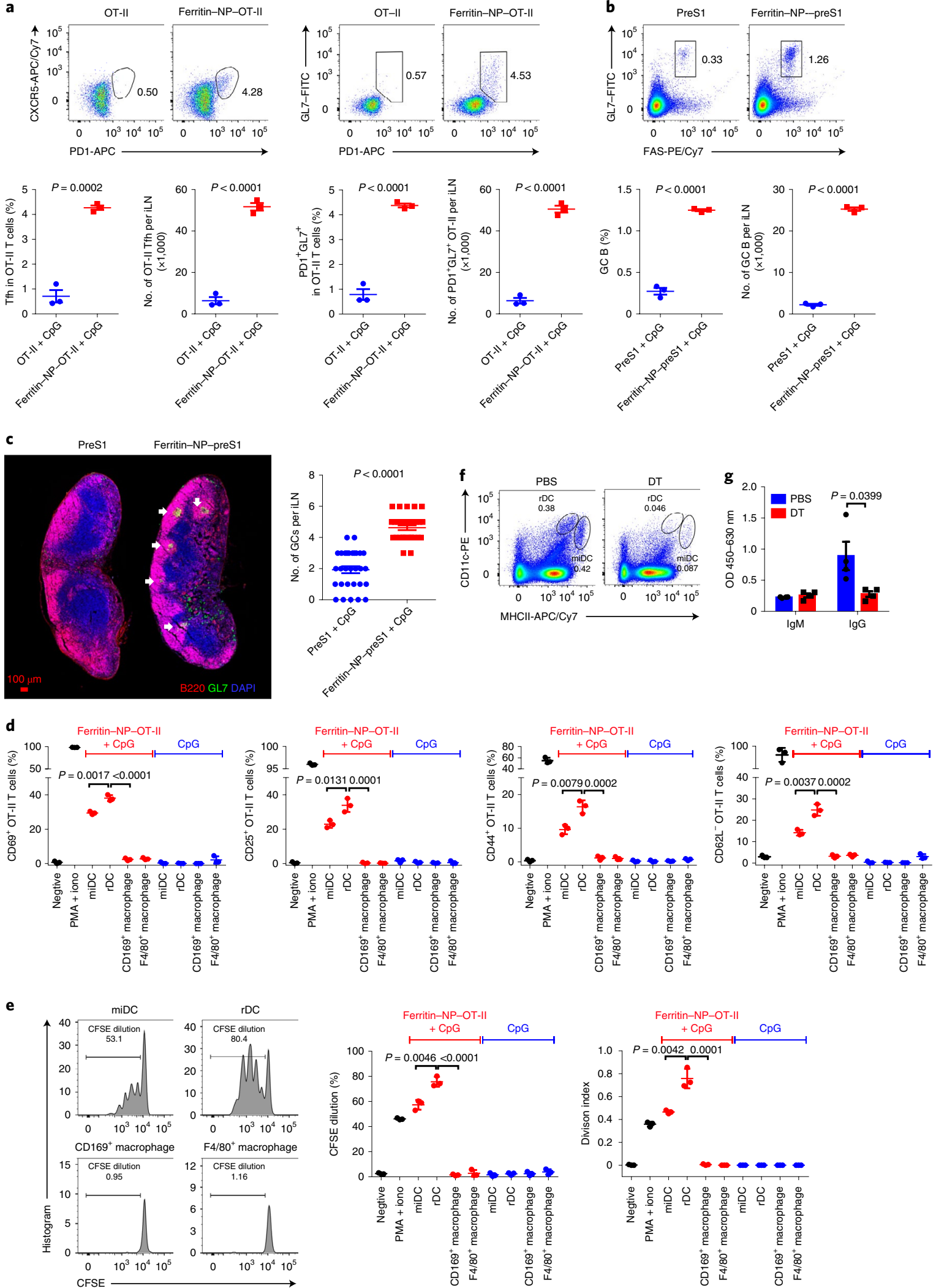
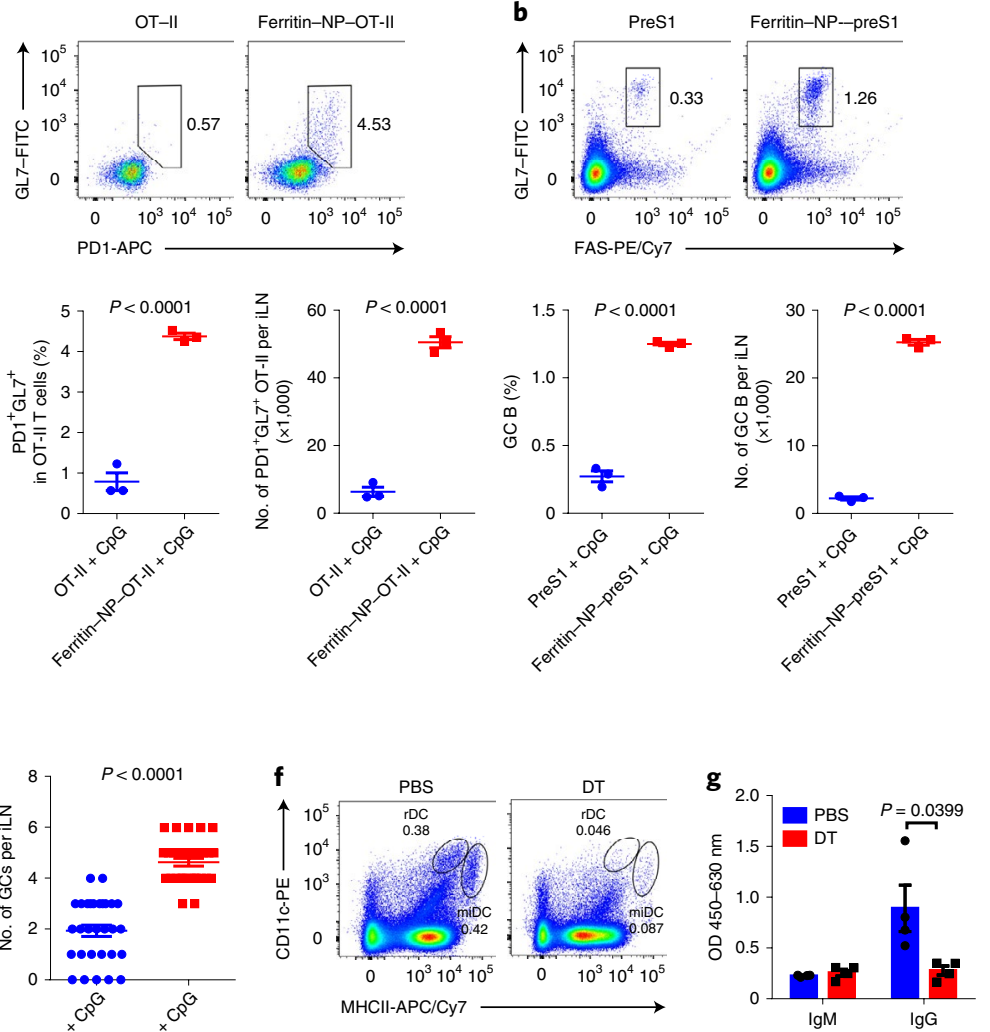
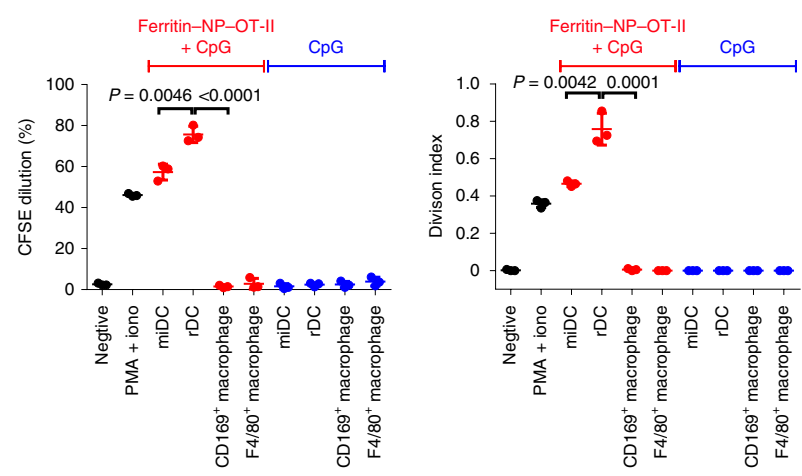

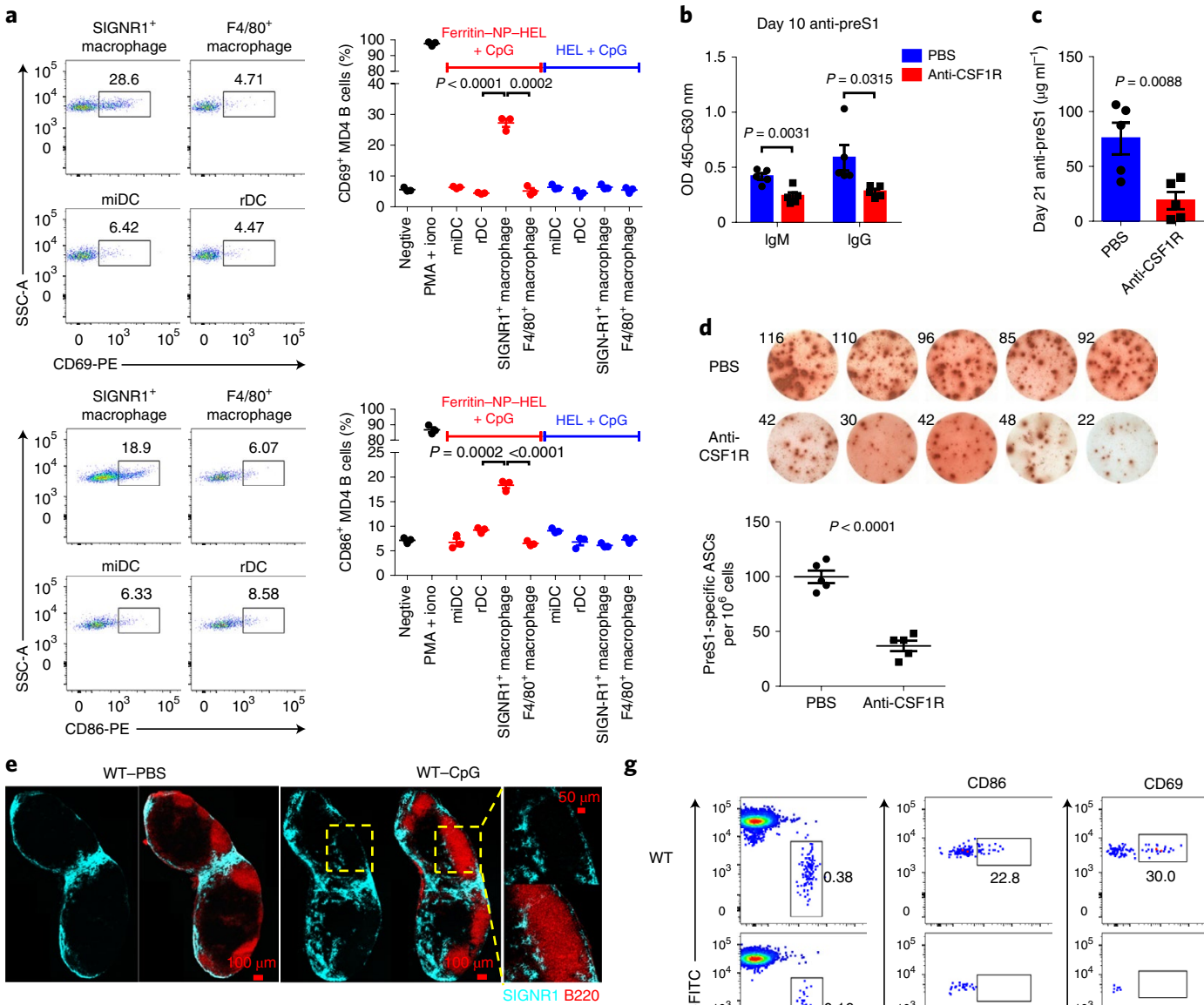

g

$\mathrm{CxCr}^{-1-}$-PBS
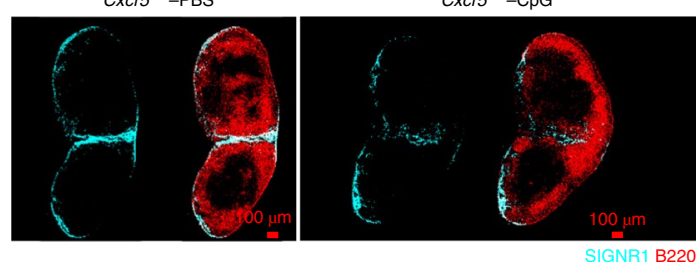

$\mathbf{f}$

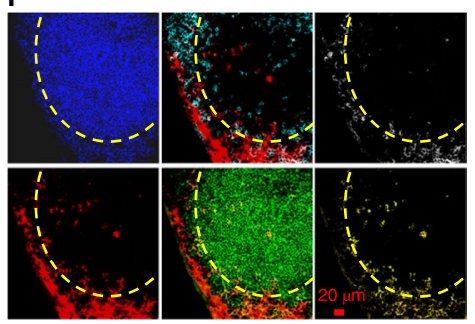

B220 SIGNR1 CD45.1CD45.2

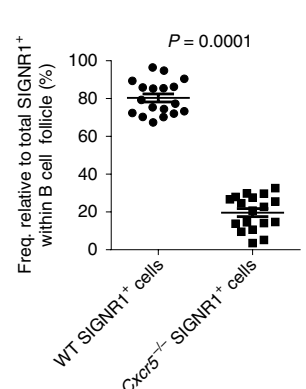

wT
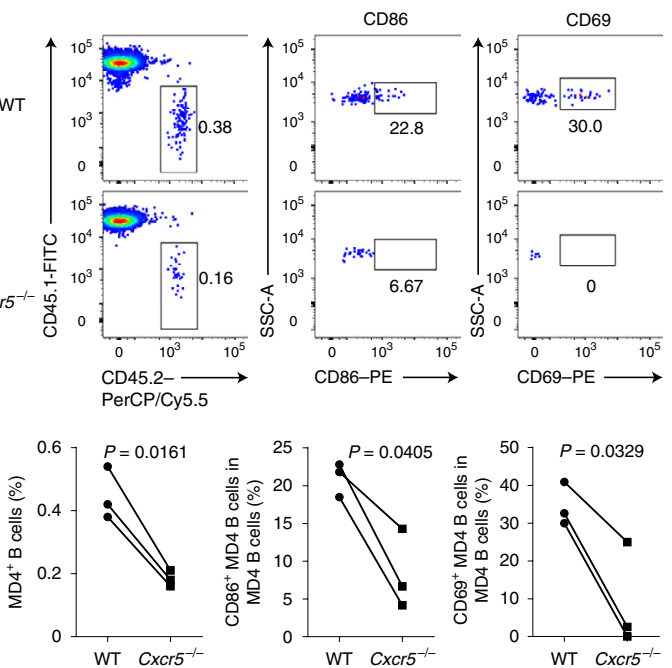

Fig. 6 | SIGNR1+ macrophages are required for B cell activation and antibody production. a, On day 3 at f.p. immunization in WT mice, rDCs, miDCs, SIGNR1+ macrophages and F4/80+ macrophages were sorted from pLNs and incubated with MD4 B cells ( $n=3$ culture wells). Activation of MD4 B cells was detected $24 \mathrm{~h}$ later. Data are representative results of four independent experiments. b-d, WT mice ( $n=5$ ) were treated with PBS or anti-CSF1R before ferritin NP-preS1 vaccine immunization. b, On day 10, preS1-specific IgM and IgG were detected; c,d, on day 21, preS1-specific IgG (c) and ASCs (d) were detected. $\mathbf{b}-\mathbf{d}$ show representative results of two independent experiments. e, Distribution of SIGNR1+ cells in iLNs of WT and Cxcr5 ${ }^{-/-}$mice on day 3 at immunization. A representative $B$ cell follicular region is shown in magnification (top right). Data show representative results of four independent experiments. f, Microscopy of WT SIGNR1 ${ }^{+}$cell (CD45.1) or Cxcr5 ${ }^{-/-}$SIGNR1 $1^{+}$cell (CD45.2) distribution in iLNs 5 d after CpG-1826 immunization in WT:Cxcr5 $5^{-1-}$ mixed bone marrow chimaeric mice. The area of total SIGNR1+ cells (red), WT SIGNR1+ cells (yellow) or Cxcr5 ${ }^{-1-}$ SIGNR1+cells (white) within follicles (yellow dashed line) was analysed using ImageJ software. Data were pooled from $n=18$ follicles from six mice in three independent experiments. Each symbol represents an individual follicle. $\mathbf{g}$, SIGNR1+ macrophages were sorted from the spleen of vaccinated WT or Cxcr5 ${ }^{-\prime-}$ mice, injected f.p. in naive CD45.1 mice, who had received MD4 B cells (CD45.2) the day before. 1d later, the percentage and activation of CD45.2 MD4 B cells were analysed. Data show representative results of three independent experiments. PerCP, peridinin-chlorophyll-protein; PE, phycoerythrin. In a-d and $\mathbf{f}$, data are shown as mean \pm s.e.m.; statistical significance was determined with an unpaired two-tailed $t$-test. In $\mathbf{g}$, the data were analysed using a paired two-tailed $t$-test. 
its further improvement. In the current study, we have revealed that ferritin NPs are primarily captured by SIGNR1 ${ }^{+}$cells at the lymphatic zone of the dLNs, where both resident DCs and macrophages are enriched ${ }^{19,44,45}$. The ferritin-capturing DCs and macrophages play different roles in antibody response. Resident DCs appear to be the most important APCs for Tfh induction, which is also supported by the recent finding that lymphatic-sinus-associated resident DCs are strategically localized to induce rapid $\mathrm{T}$ cell response on particulate antigen immunization ${ }^{45}$.

The role of LN macrophages in antibody response has been an intriguing question. In the previous studies ${ }^{34,46,47}$, subcapsular sinus macrophages were considered immotile and the particulate antigen was speculated to be transferred to B cells via transcytosis or cytoplasmic membrane movement from the subcapsular sinus side to the other side facing the B cell follicles ${ }^{19,46,48}$. In our study, we found that SIGNR1 ${ }^{+}$macrophages, but not other macrophages or DCs, can upregulate CXCR5 on immunization and migrate to the follicular regions. When CXCR5 is lacking, antigen-loaded macrophages fail to activate $B$ cells, which might suggest a previously unrecognized means of particulate antigen transfer by migrating SIGNR $1^{+}$macrophages. Whether CXCR5 deficiency could impair other functions of macrophages, resulting in impaired $B$ cell activation, remains to be studied in future. It should be noted that the upregulation of CXCR5 on macrophages is dependent on CpG instead of ferritin NPs themelves. How CpG stimulation upregulates macrophage CXCR5 expression is still unknown. Whether other adjuvants have similar effects also remains to be systemically investigated. Nonetheless, ferritin NPs seem to be able to employ this machinery for enhanced antibody response.

In summary, data presented here demonstrated that a ferritinNP-based preS1 vaccine manifests an efficient antibody response that is both preventive and therapeutic in a mouse HBV model via coordinately dual-targeting SIGNR $^{+}$resident $\mathrm{DCs}$ and macrophages in the LNs. This study might provide a good candidate and offer new insights for future translational study on CHB treatment.

\section{Online content}

Any methods, additional references, Nature Research reporting summaries, source data, extended data, supplementary information, acknowledgements, peer review information; details of author contributions and competing interests; and statements of data and code availability are available at https://doi.org/10.1038/s41565020-0648-y.

Received: 12 November 2018; Accepted: 24 January 2020; Published online: 2 March 2020

\section{References}

1. Guidotti, L. G. \& Chisari, F. V. Immunobiology and pathogenesis of viral hepatitis. Annu Rev. Pathol. 1, 23-61 (2006).

2. Gerlich, W. H. Prophylactic vaccination against hepatitis B: achievements, challenges and perspectives. Med. Microbiol. Immunol. 204, 39-55 (2015).

3. Dembek, C., Protzer, U. \& Roggendorf, M. Overcoming immune tolerance in chronic hepatitis B by therapeutic vaccination. Curr. Opin. Virol. 30, 58-67 (2018).

4. Glebe, D. et al. Pre-S1 antigen-dependent infection of Tupaia hepatocyte cultures with human hepatitis B virus. J. Virol. 77, 9511-9521 (2003).

5. Cai, D. et al. Identification of disubstituted sulfonamide compounds as specific inhibitors of hepatitis B virus covalently closed circular DNA formation. Antimicrob. Agents Chemother. 56, 4277-4288 (2012).

6. Maeng, C. Y., Ryu, C. J., Gripon, P., Guguen-Guillouzo, C. \& Hong, H. J. Fine mapping of virus-neutralizing epitopes on hepatitis B virus PreS1. Virology 270, 9-16 (2000)

7. Chen, X., Li, M., Le, X., Ma, W. \& Zhou, B. Recombinant hepatitis B core antigen carrying preS1 epitopes induce immune response against chronic HBV infection. Vaccine 22, 439-446 (2004).

8. Li, D. et al. A potent human neutralizing antibody Fc-dependently reduces established HBV infections. eLife 6, e26738 (2017).
9. Coursaget, P. et al. Antibody response to preS1 in hepatitis-B-virus-induced liver disease and after immunization. Res. Virol. 141, 563-570 (1990).

10. Deepen, R., Heermann, K. H., Uy, A., Thomssen, R. \& Gerlich, W. H. Assay of preS epitopes and preS1 antibody in hepatitis B virus carriers and immune persons. Med. Microbiol. Immunol. 179, 49-60 (1990).

11. Bian, Y. et al. Vaccines targeting preS1 domain overcome immune tolerance in hepatitis B virus carrier mice. Hepatology 66, 1067-1082 (2017).

12. Heermann, K. H. et al. Large surface proteins of hepatitis B virus containing the pre-s sequence. J. Virol. 52, 396-402 (1984).

13. Ganem, D. \& Prince, A. M. Hepatitis B virus infection-natural history and clinical consequences. N. Engl. J. Med. 350, 1118-1129 (2004).

14. Park, J.-h, Cho, E.-w, Lee, Y.-j, Shin, S. Y. \& Kim, K. L. Determination of the protective effects of neutralizing anti-hepatitis B virus (HBV) immunoglobulins by epitope mapping with recombinant HBV surface-antigen proteins. Microbiol. Immunol. 44, 703-710 (2000).

15. Yang, D. et al. A mouse model for HBV immunotolerance and immunotherapy. Cell. Mol. Immunol. 11, 71-78 (2014).

16. Smith, D. M., Simon, J. K. \& Baker, J. R. Jr. Applications of nanotechnology for immunology. Nat. Rev. Immunol. 13, 592-605 (2013).

17. Clarke, B. E. et al. Improved immunogenicity of a peptide epitope after fusion to hepatitis B core protein. Nature 330, 381-384 (1987).

18. Mildner, A. \& Jung, S. Development and function of dendritic cell subsets. Immunity 40, 642-656 (2014).

19. Gray, E. E. \& Cyster, J. G. Lymph node macrophages. J. Innate Immun. 4, 424-436 (2012).

20. Kanekiyo, M. et al. Self-assembling influenza nanoparticle vaccines elicit broadly neutralizing H1N1 antibodies. Nature 499, 102-106 (2013).

21. Yassine, H. M. et al. Hemagglutinin-stem nanoparticles generate heterosubtypic influenza protection. Nat. Med. 21, 1065-1070 (2015).

22. Kanekiyo, M. et al. Rational design of an Epstein-Barr virus vaccine targeting the receptor-binding site. Cell 162, 1090-1100 (2015).

23. $\mathrm{Bu}$, W. et al. Immunization with components of the viral fusion apparatus elicits antibodies that neutralize Epstein-Barr virus in B cells and epithelial cells. Immunity 50, 1305-1316.e1306 (2019).

24. Kanekiyo, M. et al. Mosaic nanoparticle display of diverse influenza virus hemagglutinins elicits broad B cell responses. Nat. Immunol. 20, 362-372 (2019)

25. Zakeri, B. et al. Peptide tag forming a rapid covalent bond to a protein, through engineering a bacterial adhesin. Proc. Natl Acad. Sci. USA 109, E690-E697 (2012).

26. Liu, Z. et al. A novel method for synthetic vaccine construction based on protein assembly. Sci. Rep. 4, 7266 (2014).

27. Tatur, J., Hagedoorn, P. L., Overeijnder, M. L. \& Hagen, W. R. A highly thermostable ferritin from the hyperthermophilic archaeal anaerobe Pyrococcus furiosus. Extremophiles 10, 139-148 (2006).

28. Yan, H. et al. Sodium taurocholate cotransporting polypeptide is a functional receptor for human hepatitis B and D virus. eLife 1, e00049 (2012).

29. Pontisso, P. et al. Identification of an attachment site for human liver plasma membranes on hepatitis B virus particles. Virology 173, 522-530 (1989)

30. Mimms, L. T. et al. Discrimination of hepatitis B virus (HBV) subtypes using monoclonal antibodies to the PreS1 and PreS2 domains of the viral envelope. Virology 176, 604-619 (1990).

31. Ivashkiv, L. B. IFN $\gamma$ : signalling, epigenetics and roles in immunity, metabolism, disease and cancer immunotherapy. Nat. Rev. Immunol. 18, 545-558 (2018).

32. Gonzalez, S. F. et al. Capture of influenza by medullary dendritic cells via SIGN-R1 is essential for humoral immunity in draining lymph nodes. Nat. Immunol. 11, 427-434 (2010).

33. Conde, P. et al. DC-SIGN ${ }^{+}$macrophages control the induction of transplantation tolerance. Immunity 42, 1143-1158 (2015).

34. Junt, T. et al. Subcapsular sinus macrophages in lymph nodes clear lymph-borne viruses and present them to antiviral B cells. Nature 450, 110-114 (2007).

35. Park, C. G. et al. Five mouse homologues of the human dendritic cell C-type lectin, DC-SIGN. Int. Immunol. 13, 1283-1290 (2001).

36. Powlesland, A. S. et al. Widely divergent biochemical properties of the complete set of mouse DC-SIGN-related proteins. J. Biol. Chem. 281, 20440-20449 (2006)

37. Parent, S. A. et al. Molecular characterization of the murine SIGNR1 gene encoding a C-type lectin homologous to human DC-SIGN and DC-SIGNR. Gene 293, 33-46 (2002)

38. Angel, C. E. et al. Distinctive localization of antigen-presenting cells in human lymph nodes. Blood 113, 1257-1267 (2009).

39. Vinuesa, C. G., Linterman, M. A., Yu, D. \& MacLennan, I. C. Follicular helper T cells. Annu Rev. Immunol. 34, 335-368 (2016).

40. Cyster, J. G. et al. Follicular stromal cells and lymphocyte homing to follicles. Immunological Rev. 176, 181-193 (2000).

41. Ning, Q. et al. Roadmap to functional cure of chronic hepatitis B: an expert consensus. J. Viral Hepat. 26, 1146-1155 (2019). 
42. Tang, L. S. Y., Covert, E., Wilson, E. \& Kottilil, S. Chronic hepatitis B infection: a review. J. Am. Med. Assoc. 319, 1802-1813 (2018).

43. Revill, P. A. et al. A global scientific strategy to cure hepatitis B. Lancet Gastroenterol. Hepatol. 4, 545-558 (2019).

44. Gerner, M. Y., Kastenmuller, W., Ifrim, I., Kabat, J. \& Germain, R. N. Histo-cytometry: a method for highly multiplex quantitative tissue imaging analysis applied to dendritic cell subset microanatomy in lymph nodes. Immunity 37, 364-376 (2012).

45. Gerner, M. Y., Torabi-Parizi, P. \& Germain, R. N. Strategically localized dendritic cells promote rapid $\mathrm{T}$ cell responses to lymph-borne particulate antigens. Immunity 42, 172-185 (2015)
46. Phan, T. G., Green, J. A., Gray, E. E., Xu, Y. \& Cyster, J. G. Immune complex relay by subcapsular sinus macrophages and noncognate B cells drives antibody affinity maturation. Nat. Immunol. 10, 786-793 (2009).

47. Phan, T. G., Grigorova, I., Okada, T. \& Cyster, J. G. Subcapsular encounter and complement-dependent transport of immune complexes by lymph node B cells. Nat. Immunol. 8, 992-1000 (2007).

48. Cyster, J. G. B cell follicles and antigen encounters of the third kind. Nat. Immunol. 11, 989-996 (2010).

Publisher's note Springer Nature remains neutral with regard to jurisdictional claims in published maps and institutional affiliations.

( $)$ The Author(s), under exclusive licence to Springer Nature Limited 2020 


\section{Methods}

Mice. Naïve WT C57BL/6 mice and BALB/c mice were obtained from Vital River. CD11c-DTR mice and OT-II TCR transgenic mice were purchased from The Jackson Laboratory. Signr1 $1^{-/}$mice were generated by Cyagen. MD4 transgenic mice were provided by B. Hou (Chinese Academy of Science Key Laboratory for Infection and Immunity, Institute of Biophysics, Chinese Academy of Sciences, Beijing, China). Cxcr5 $5^{-1-}$ mice were kindly provided by H. Qi (Institute for Immunology, School of Medicine, Tsinghua University, Beijing, China). All mice were housed under specific pathogen-free conditions in the animal care facilities at the Institute of Biophysics, Chinese Academy of Sciences. All animal experiments were performed in accordance with the guidelines of the Institute of Biophysics, Chinese Academy of Sciences, using protocols approved by the institutional laboratory animal care and use committee.

Human sample. Human fresh distal mesenteric LNs from neuroblastoma patients 2.5-14 years old were derived from the patients who underwent surgery at the Department of Paediatric Surgical Oncology, Children's Hospital of Chongqing Medical University. The study was performed in accordance with the committee guidelines and regulations. Informed consent was obtained from all patients.

Cloning, expression and purification of fusion proteins. The gene coding for $P f$ ferritin (residues 5-174) was synthesized by GENEWIZ using E. coli preferred codons. SpyTag spaced with three G4S linkers was fused to the amino-terminus of $P f$ ferritin during PCR. The SpyTag-Pf ferritin gene was cloned into the pDEST14 prokaryotic expression plasmid using the NdeI and SacI sites. The SpyTag-mouse ferritin gene was cloned into pDEST14 in the same manner. For the cloning of $\Delta \mathrm{N}$ SC-preS1, SC-eGFP, SC-OT-II and SC-HEL, the PCR product of SC- $\left(\mathrm{G}_{4} \mathrm{~S}\right)_{3}$ was cloned into pDEST14 using the NdeI and EcoRI sites and the antigen gene was cloned using the EcoRI and SacI sites. For the cloning of preS1 without SC, preS1 was cloned into pDEST14 using the NdeI and SacI sites. The preS1 sequence consisting of the first 108 amino acids (ayw) of HBV large surface protein was synthesized by PCR from complementary DNA extracted from livers of mice infected with AAV-HBV1.3 (genotype D3, subtype ayw3). The pDEST14-SC-Pf ferritin clone was obtained in the same manner.

For SpyTag-Pfferritin NP expression and purification, BL21 (DE3) competent E. coli cells were transformed with pDEST14-SpyTag- $\left(\mathrm{G}_{4} \mathrm{~S}\right)_{3}-P f$ ferritin plasmid, and a single colony was picked and cultured in $5 \mathrm{ml}$ lysogeny broth at $37^{\circ} \mathrm{C}$ overnight. The cultures were then amplified in $400 \mathrm{ml}$ lysogeny broth and induced by $0.3 \mathrm{mM}$ isopropylthiogalactoside for $6 \mathrm{~h}$. The bacterial cultures were harvested and lysed in Tris buffer $(20 \mathrm{mM}$ Tris, $50 \mathrm{mM} \mathrm{NaCl}, \mathrm{pH} 7.5)$. The lysate supernatants were diluted to $1 \mathrm{mg} \mathrm{ml}^{-1}$ and heated to $70^{\circ} \mathrm{C}$ for $15 \mathrm{~min}$ to precipitate most of the E. coli proteins. After centrifugation and concentration, the supernatants were loaded onto a Superose 6 Increase (GE Healthcare) size exclusion column that was pre-equilibrated with $20 \mathrm{mM}$ Tris $50 \mathrm{mM} \mathrm{NaCl}$ buffer ( $\mathrm{pH} 7.5$ ) and eluted with the same buffer at a rate of $0.5 \mathrm{ml} \mathrm{min}^{-1}$. The total volume of the column $\left(V_{\mathrm{t}}\right)$ was $24 \mathrm{ml}$, the outer volume of the separation medium $\left(V_{\mathrm{o}}\right)$ was $8 \mathrm{ml}$ and the elution volume $\left(V_{\mathrm{e}}\right)$ of SpyTagferritin NP was $15 \mathrm{ml}$. The expression and purification of SpyTag-mouse ferritin NPs and SC-Pf ferritin NPs were the same as for SpyTag- $P f$ ferritin NPs. The expression of SC-preS1 was the same as for SpyTag-Pfferritin NPs. For SC-preS1 purification, the lysate supernatants were purified using a Ni-NTA agarose column (ComWin Biotech) according to the manufacturer's protocol. SC-eGFP, SC-OT-II, SC-HEL and preS1 without SC were expressed and purified in the same manner as SC-preS1. Endotoxin was removed from all of the proteins with a ToxinEraser endotoxin removal kit (GenScript). The amount of endotoxin is less than $0.1 \mathrm{EU} \mathrm{ml}^{-1}$.

Generation and purification of ferritin-NP-based vaccine. The purified SC-preS1 was conjugated to SpyTag-ferritin NP in vitro to construct the ferritin NP-preS1 vaccine. To assay reconstitution, SpyTag-ferritin NP was reacted with SC-preS1 at molar ratios of 1:0.5, 1:1 and $1: 2$ at $4^{\circ} \mathrm{C}$ overnight. SDS-PAGE was used to evaluate the reconstitution efficiency. For vaccine generation, SpyTagferritin NP was mixed with SC-preS1 at a molar ratio of $1: 1.5$ at $4{ }^{\circ} \mathrm{C}$ overnight. The conjugated ferritin NP-preS1 was then purified using a Superose 6 Increase size exclusion column to remove the unbound SC-preS1. Ferritin NP-preS1 was eluted in $11.5 \mathrm{ml}\left(V_{\mathrm{t}}=24 \mathrm{ml}, V_{\mathrm{o}}=8 \mathrm{ml}\right)$. The generation and purification of mouse ferritin NP-preS1, ferritin NP-eGFP, ferritin NP-OT-II and ferritin NP-HEL were performed in the same manner. To abrogate reconstitution in the specific assay, SC-ferritin NP and SC-preS1 were mixed physically to avoid their interaction. In addition, when the $\mathrm{pH}$ of the reaction buffer reached 11.5 , the combination between SC and SpyTag was almost lost. Endotoxin was removed from the vaccine proteins with the ToxinEraser endotoxin removal kit before immunization. The amount of endotoxin is less than $0.1 \mathrm{EU} \mathrm{ml}^{-1}$.

Negative-stain electron microscopy. Grids of ferritin NPs for negative-stain electron microscopy were prepared as described previously ${ }^{49}$. Briefly, $4 \mu \mathrm{l}$ samples $\left(0.15 \mathrm{mg} \mathrm{ml}^{-1}\right)$ were applied to glow-discharge electron microscopy grids covered by a thin layer of continuous carbon film and stained with $2 \%$ (w/v) uranyl acetate. Negatively stained grids were imaged on a Talos L120C microscope (Thermo Fisher) operating at $120 \mathrm{kV}$. Images were recorded at a magnification of $\times 12,000$ and a defocus set to $-2 \mu \mathrm{m}$, using a $4 \mathrm{k} \times 4 \mathrm{k}$ CCD (charge-coupled device) camera.
The particles were manually picked and two-dimensional classification was performed with EMAN2.

Immunization. Female naïve WT C57BL/6 mice or BALB/c mice (8-9 weeks old) were subcutaneously immunized with $500 \mathrm{pmol}$ (approximately $23.9 \mu \mathrm{g}$, as determined by single ferritin-preS1 subunit) ferritin NP-preS1 vaccine or $500 \mathrm{pmol}$ (approximately $12.7 \mu \mathrm{g}$ ) SC-preS1 with $30 \mu \mathrm{g}$ CpG-1826 (Generay), respectively, at the tail base at day 0 and day 14 . For the memory response assay, another boost immunization was performed at day 270 . For high-dose immunization, $4 \mathrm{nmol}$ (approximately $191.3 \mu \mathrm{g}$ ) ferritin NP-preS1 vaccine or $4 \mathrm{nmol}$ (approximately $101.8 \mu \mathrm{g}) \mathrm{SC}-$ preS1 with $30 \mu \mathrm{g}$ CpG-1826 was used. For the HBV prevention assay in vivo, $200 \mathrm{pmol}$ (approximately $9.6 \mu \mathrm{g}$ ) ferritin NP-preS1 vaccine or

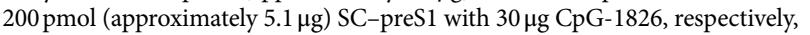
was administered. For the therapy assay in vivo, $500 \mathrm{pmol}$ (approximately $23.9 \mu \mathrm{g}$ ) ferritin NP-preS1 vaccine or 500 pmol (approximately $12.7 \mu \mathrm{g}$ ) SC-preS1 with $30 \mu \mathrm{g}$ CpG-1826 was administered four times on days 35, 49, 63 and 77. Mouse ferritin $\mathrm{NP}-$ preS1 vaccine and other soluble proteins were immunized with the indicated dose in the same manner. IFN- $\gamma$ blockade was performed using an intraperitoneal injection of $200 \mu \mathrm{g}$ anti-IFN- $\gamma$ (XMG1.2, BioXcell) at indicated times.

ELISA. For the preS1-specific ELISA, $5 \mu \mathrm{g} \mathrm{ml}^{-1}$ preS1 protein produced in our laboratory was coated onto 96-well high-binding Costar assay plates (Corning) at $4{ }^{\circ} \mathrm{C}$ overnight. After blocking with a blocking buffer (PBS containing $5 \%$ fetal bovine serum, FBS), serum samples with different dilutions (1:10-1:10000) were added to the plates. Horseradish peroxidase (HRP)-conjugated goat anti-mouse $\operatorname{IgG}(\mathrm{H}+\mathrm{L})(1: 5,000, \mathrm{ZSGB}-\mathrm{BIO})$, goat anti-mouse IgG1 (1:5,000, ProteinTech), goat anti-mouse IgG2c (1:5,000, ProteinTech) and goat anti-mouse IgM (1:5,000, ProteinTech) were used to detect each isotype. The concentration of specific antibodies was measured using TMB substrate (SeraCare) and the absorbance at $450 \mathrm{~nm}-630 \mathrm{~nm}$ was detected using a microplate reader (Molecular Devices). The absolute concentration of anti-preS1 IgG in serum is calibrated using a mouse monoclonal anti-Hep B preS1 (KR127, Santa Cruz) standard. For the detection of anti-preS1 avidity, mouse sera were serially diluted on the basis of IgG concentration and drawn on the $x$ axis, and anti-preS1 levels of diluted sera were measured and drawn on the $y$ axis simultaneously. The slope of the fitting curve was calculated to reflect the avidity of the antisera. For the detection of mouse ferritin- or Pf ferritin-specific antibody, plates were coated with $5 \mu \mathrm{g} \mathrm{ml}^{-1}$ mouse ferritin or $P f$ ferritin protein produced in our laboratory, and goat anti-mouse IgG $(\mathrm{H}+\mathrm{L})-\mathrm{HRP}$ antibodies were used as detection antibodies. For the ELISA to test preS1 antigen located on HBsAg or HBV particles, plates were coated with $5 \mu \mathrm{g} \mathrm{ml}^{-1}$ anti-preS1 monoclonal antibody XY007 (provided by Y.-X. Fu), and the detection antibody was anti-HBs-HRP (Shanghai Kehua Bio-engineering). For HBsAg, HBeAg and antiHBs testing, commercial ELISA kits were used (Shanghai Kehua Bio-engineering).

ELISpot. For IgG ASC detection, $10 \mu \mathrm{g} \mathrm{ml}^{-1}$ preS1 protein was coated onto 96-well ELISpot plates (Merck Millipore) at $4^{\circ} \mathrm{C}$ overnight. After blocking with a blocking buffer (RPMI 1640 medium containing $10 \%$ FBS), $1 \times 10^{6}$ lymphocytes from LNs in $100 \mu \mathrm{l}$ complete RPMI 1640 medium were added to the plates. After a 5-6h incubation at $37^{\circ} \mathrm{C}$, preS1-specific IgG ASCs were analysed using a biotinylated donkey anti-mouse IgG (CWbio) and streptavidin-HRP (BD Biosciences). The spots were visualized with an AEC substrate set (BD Biosciences) and quantified with an autoanalysis system. For IFN- $\gamma$-secreting cell detection, $5 \times 10^{5}$ lymphocytes isolated from LNs, spleen and livers were incubated for $48 \mathrm{~h}$ at $37^{\circ} \mathrm{C}$ in complete RPMI 1640 medium containing $5 \mu \mathrm{g} \mathrm{ml}^{-1}$ full preS1 protein or control protein in an IFN- $\gamma$ ELISpot plate (Merck Millipore).

AAV-HBV1.3 infection. The AAV-HBV1.3 virus was purchased from the Beijing FivePlus Molecular Medicine Institute. This recombinant virus carries 1.3 copies of the HBV genome (genotype D3, subtype ayw3) and is packaged in AAV serotype 8 capsids $^{15}$. Male adult C57BL/6 mice were injected with $1 \times 10^{10} \mathrm{vg}$ recombinant virus in $100 \mu \mathrm{l}$ saline by tail vein injection. For the therapeutic assay, stable HBV carrier mice were used for vaccination after 5 weeks. Mice were bled through the ophthalmic vein at the indicated times in the respective experiments to monitor preS1 antibody, HBsAg, preS1 antigen and HBV genomic DNA in serum.

HBV infection and inhibition assays in vitro. The HepG2-hNTCP cell line and HBV (genotype D3, subtype ayw3) virus were provided by W. Li. In vitro $\mathrm{HBV}$ infection and inhibition assays were performed as reported ${ }^{11,28}$. Briefly, $1 \times 10^{7}$ copies of genome-equivalent HBV were inoculated into the culture medium of $1 \times 10^{5} \mathrm{HepG} 2$-hNTCP cells in 48 -well plates in the presence of antipreS1 sera from different mice and incubated for $24 \mathrm{~h}$. The cells were then washed with medium three times and maintained in primary hepatocyte maintenance medium (William's E medium (Gibco) with $5 \mu \mathrm{g} \mathrm{ml}^{-1}$ transferrin, $5 \mathrm{ng} \mathrm{ml}^{-1}$ sodium selenite, $3 \mu \mathrm{g} \mathrm{ml}^{-1}$ insulin (insulin-transferrin-sodium selenite; Corning),

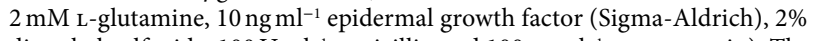
dimethyl sulfoxide, $100 \mathrm{U} \mathrm{ml}^{-1}$ penicillin and $100 \mu \mathrm{g} \mathrm{ml}^{-1}$ streptomycin). The medium was changed every 2 days. Viral infection at different times was analysed by measuring HBsAg and HBeAg in culture medium and detecting HBV RNAs in HepG2-hNTCP cells. 
Bone marrow chimaera. C57BL/6 mice were lethally irradiated with Co60 at $10 \mathrm{~Gy}$. The next day, $5 \times 10^{6}$ bone marrow cells from donor mice (CD11c-DTR mice or CXcr5 $5^{-/-}$mice) were intravenously transferred. Mixed bone marrow chimaeras were generated by transplanting an equal number of $2.5 \times 10^{6} \mathrm{WT}$ CD 45.1 and $2.5 \times 10^{6}$ Cxcr $5^{-1-}$ CD 45.2 bone marrow cells. The chimaeras were given prophylactic water containing antibiotics for 4 weeks following irradiation. Then, 8-10 weeks after bone marrow transplantation, the mice could be used for subsequent operations.

DC and macrophage depletion. For systemic DC depletion, 8-10 weeks after CD11c-DTR bone marrow transfer, the chimaeric mice were given DT (Sigma) intraperitoneally at a dose of $5 \mathrm{ng}$ per $\mathrm{g}$ body weight $24 \mathrm{~h}$ before deletion efficiency detection or vaccine immunization. To maintain the depletion, DT was administered every two days.

For macrophage depletion in pLN, C57BL/6 mice were injected with 30-50 $\mu \mathrm{l}$ CLLs (FormuMax) or control liposomes (CON) f.p. 7-10 d before experimentation.

For systemic macrophage depletion, a blocking antibody to CSF1R (clone ASF98, BioXCell) was administered. $50 \mu \mathrm{g}$ anti-CSF1R was injected via f.p. and $100 \mu \mathrm{g}$ via intraperitoneal injection once a day for three consecutive days before experimentation.

LN digestion and flow cytometry. LNs were harvested and gently minced using scissors in FACS buffer (PBS containing 2\% FBS). LNs were then digested with $0.5 \mathrm{mg} \mathrm{ml}^{-1}$ collagenase I (Sigma) and $0.04 \mathrm{mg} \mathrm{ml}^{-1} \mathrm{DNase} \mathrm{I}$ (Roche) at $37^{\circ} \mathrm{C}$ for $1 \mathrm{~h}$. For CXCR5 detection and in the ferritin-FITC binding assay, since CXCR 5 and FITC were highly susceptible to collagenase digestion, CLSPA (Worthington) was used. LNs were digested with $100 \mathrm{U} \mathrm{ml}^{-1}$ CLSPA and $0.04 \mathrm{mg} \mathrm{ml}^{-1} \mathrm{DNase}$ I (Roche) in 1640 medium at $37^{\circ} \mathrm{C}$ for $1 \mathrm{~h}$. The suspension was washed with cold FACS buffer, disaggregated by passing through a $70-\mu \mathrm{m}$ cell strainer (Biologix Group) and centrifuged at $500 \mathrm{~g}$ for $5 \mathrm{~min}$. Splenocytes were collected in the same manner, and red blood cells were removed with ammonium-chloride-potassium buffer.

For flow cytometric analysis, single cells were resuspended in an appropriate volume of FACS buffer $\left(1-5 \times 10^{6}\right.$ cells per $\left.100 \mu \mathrm{l}\right)$, blocked with anti-Fc $\gamma \mathrm{R}$ monoclonal antibody (clone 2.4G2) to block non-specific binding, and labelled with fluorescence-conjugated antibodies. For DC and macrophage analysis, the antibodies used included anti-CD19 (6D5, BioLegend), anti-B220 (RA3-6B2, eBioscience), anti-CD11c (N418, BioLegend), anti-I-A/I-E (M5/114.15.2, BioLegend), anti-CD11b (M1/70, eBioscience), anti-SIGNR1 (22D1, eBioscience), anti-CD169 (3D6.112, BioLegend), anti-F4/80 (BM8, BioLegend) and anti-CD103 (2E7, eBioscience). For $\mathrm{T}$ cell and $\mathrm{B}$ cell activation analysis, the antibodies used included anti-Thy1.1 (HIS51, eBioscience), anti-TCR V $\alpha 2$ (B20.1, eBioscience), anti-CD4 (GK1.5, BioLegend), anti-PD1 (J43, eBioscience), anti-CXCR5 (SPRCL5, eBioscience), anti-GL7 (GL-7, eBioscience), anti-FAS (Jo2, BD), anti-CD69 (H1.2F3, eBioscience), anti-CD25 (PC61.5, eBioscience), anti-CD44 (IM7, BioLegend), anti-CD62L (MEL-14, eBioscience) and anti-CD86 (GL1, eBioscience). The working concentration of the antibodies is $2.5 \mu \mathrm{g} \mathrm{ml}^{-1}$. For human immune cell staining, the antibodies used included Human Trustain FcX (BioLegend), anti-human CD14 (M5E2, BioLegend), anti-human HLA-DR (L243, BioLegend) and anti-human CD209 (9E9A8, BioLegend). For the T cell proliferation assay, OT-II T cells were labelled with $2 \mathrm{mM}$ carboxyfluorescein succinimidyl ester (Sigma) in PBS for $10 \mathrm{~min}$ at $37^{\circ} \mathrm{C}$, then washed with PBS containing 10\% FBS at least twice. An LSRFortessa flow cytometer (BD Biosciences) and FlowJo software (Tree Star) were used for data collection and analysis, respectively. Cell sorting was performed on a FACSAria III (BD Biosciences)

Immunofluorescence microscopy. For the detection of ferritin NP-eGFP or FITC-labelled ferritin NPs, LNs were harvested and fixed with periodate-lysineparaformaldehyde fixative buffer (PLP, PBS buffer containing $2 \mathrm{mg} \mathrm{ml}^{-1} \mathrm{NaIO}_{4}$, $0.1 \mathrm{M} \mathrm{L}$-lysine ( $\mathrm{pH} 7.4$ ) and $1 \%(\mathrm{w} / \mathrm{v})$ PFA) for $12 \mathrm{~h}$, then dehydrated in $30 \%$ sucrose twice before embedding in optimal cutting temperature compound (Sakura) and snap frozen in liquid nitrogen. Next, $10 \mu \mathrm{m}$-thick cryosections were prepared. For GC and SIGNR1 ${ }^{+}$cell staining, LNs were embedded in optimal cutting temperature compound and snap frozen in liquid nitrogen directly. After cryosectioning, the slides were air-dried for $1 \mathrm{~h}$ and fixed for $10 \mathrm{~min}$ in cold acetone.

The cryosections were blocked for $2 \mathrm{~h}$ at room temperature in FACS buffer containing $1 \mathrm{mg} \mathrm{ml}^{-1}$ anti-Fc $\gamma \mathrm{R} \mathrm{mAb}$ (2.4G2). The cryosections were incubated overnight at $4{ }^{\circ} \mathrm{C}$ with the following antibodies at $2.5 \mu \mathrm{g} \mathrm{ml}^{-1}$ working concentrations: anti-SIGNR1 (22D1, eBioscience), anti-B220 (RA3-6B2, eBioscience), anti-CD45.1 (A20, eBioscience), anti-CD45.2 (104, eBioscience) and anti-GL7 (GL-7, eBioscience). For eGFP staining, anti-full length GFP polyclonal antibody $\left(1: 1,000\right.$, Clontech) was used overnight at $4^{\circ} \mathrm{C}$. Then, the unconjugated anti-GFP antibody was detected with an AlexaFluor488-conjugated goat antirabbit IgG secondary antibody $(1: 1,000, \mathrm{Zsbio})$ at room temperature for $1 \mathrm{~h}$. Images were taken on a confocal microscope (Zeiss LSM-710) and analysed with ZEN 2012 software (Carl Zeiss) and Fiji ImageJ.

Quantitative real-time PCR. Total RNA was extracted using TRIzol (Invitrogen) according to the manufacturer's instructions. RNA was reverse transcribed into cDNA using a RevertAid First Strand cDNA synthesis kit (Life Technologies). Quantitative real-time PCR was performed using SYBR Premix Ex Taq (Takara) on an ABI QuantStudio 7 Flex real-time PCR system. The primers used for quantitative real-time PCR are as follows: Hprt (forward, $5^{\prime}$-TCCTCCTCAGACC GCTTTT-3'; reverse, 5' -CCTGGTTCATCATCGCTAATC-3'), Lamp1 (forward, $5^{\prime}$-GTGGGAGTTGCGGTATCAAC-3'; reverse, 5' -TATTCAAGCGCACTCCTT GC-3'), Lamp2 (forward, 5' -CTAGGAGCCGTTCAGTCCAA-3'; reverse, 5' -CTT GCAGGTGAATACCCCAA-3'), Lyz1 (forward, 5'-GGACTCCTCCTGCTTTCT GT-3'; reverse, 5' -TAAACACACCCAGTCAGCCA-3'), Lyz2 (forward, 5' -GGACT CCTCCTGCTTTCTGT-3' ${ }^{\prime}$; reverse, $5^{\prime}$-AGTCAGTGCTTTGGTCTCCA-3' ), Ctsb (forward, 5' -TCTGAAGAAGCTGTGTGGCA-3'; reverse, 5' -TTGTTCCCGTG CATCAAAGG-3') and Ctsc (forward, $5^{\prime}$-CTCGGTGATGGAAGCAACAG-3'; reverse, $5^{\prime}$-CTGATAGCTGTGTGGCCTCT-3'). The $\Delta \Delta$ Ct method was used to calculate relative target gene expression to Hprt. For HBV RNA detection, the primers used are as follows: HBV $3.5 \mathrm{~kb}$ (forward, 5'-GAGTGTGGATTCGCACT CC-3'; reverse, 5'-GAGGCGAGGGAGTTCTTCT-3') and HBV total (forward, 5' -TCACCAGCACCATGCAAC-3'; reverse, 5' -AAGCCACCCAAGGCACAG-3') For HBV DNA detection in serum, HBV DNA was extracted from $200 \mu \mathrm{l}$ of serum and measured following the manufacturer's instructions (careHBV, Qiagen). The detection of HBV cccDNA was performed as reported ${ }^{50}$. After total DNA isolation from liver, a combination of digestion with XmaI and SacI restriction enzymes followed by T5 exonuclease was used to degrade HBV relaxed circular DNA and all the AAV DNA. The remaining undigested HBV cccDNA allows specific detection by quantitative real-time PCR. The primers used are forward, $5^{\prime}$-TGCACTTCGCTTCACCT-3'; reverse, 5' -AGGGGCATTTGGTGGTC-3'.

Statistical analysis. All analysis was performed using GraphPad Prism statistical software. All of the data were analysed using an unpaired two-tailed $t$-test or paired two-tailed $t$-test. The results are expressed as the mean \pm s.e.m. A value of $P<0.05$ was considered statistically significant.

Reporting Summary. Further information on research design is available in the Nature Research Reporting Summary linked to this article.

\section{Data availability}

The data that support the plots within this paper and other findings of this study are available from the corresponding author on reasonable request.

\section{References}

49. Roux, K. H. Negative-stain immunoelectron-microscopic analysis of small macromolecules of immunologic significance. Methods 10, 247-256 (1996).

50. Lucifora, J. et al. Detection of the hepatitis B virus (HBV) covalently-closedcircular DNA (cccDNA) in mice transduced with a recombinant AAV-HBV vector. Antivir. Res. 145, 14-19 (2017).

\section{Acknowledgements}

We thank H. Qi for $\mathrm{Cxcr5}^{-/-}$mice and B. Hou for MD4 transgenic mice. We thank K. Fan for technical consultation on ferritin characterization, and X. Shi for mouse breeding and healthcare services. This work was supported by grants from the Strategic Priority Research Programme of the Chinese Academy of Sciences (XDB29040202 to M.Z.), the National Natural Science Foundation of China (81991493 to Q.C.), the National Key R\&D Programme of China (2018YF1313000 and 2018YF1313004 to S.W., 2019YFA0905903 to M.Z.) and the Science and Technology Service Network Initiative Programme of the Chinese Academy of Sciences (KFJ-STS-ZDTP-062 to M.Z.).

\section{Author contributions}

W.W. and M.Z. designed the experiments and analysed the data; W.W. and X.Z. conducted most experiments; Y.B., H.P. and Y.-X.F. helped with an AAV-HBV1.3 infection mouse model and in vitro HBV infection assay; S.W. and Z.W. provided human LN samples and helped in the human immune cell targeting assay; Q.C. helped with the immunofluorescence staining; Z.G. and P.Z. performed transmission electron micrography imaging; X.Y. provided technical advice on ferritin preparation and characterization and helped in the iron measuring assay; W.L. provided the HepG2-hNTCP cell line and HBV virus and helped with the in vitro infection assay; W.W. and M.Z. wrote the manuscript; M.Z. conceived and supervised the project.

\section{Competing interests}

The authors declare no competing interests.

\section{Additional information}

Supplementary information is available for this paper at https://doi.org/10.1038/ s41565-020-0648-y.

Correspondence and requests for materials should be addressed to M.Z.

Peer review information Nature Nanotechnology thanks John Kehrl, Daniel Shouval and the other, anonymous, reviewer(s) for their contribution to the peer review of this work.

Reprints and permissions information is available at www.nature.com/reprints. 


\section{Reporting Summary}

Nature Research wishes to improve the reproducibility of the work that we publish. This form provides structure for consistency and transparency in reporting. For further information on Nature Research policies, see Authors \& Referees and the Editorial Policy Checklist.

\section{Statistical parameters}

When statistical analyses are reported, confirm that the following items are present in the relevant location (e.g. figure legend, table legend, main text, or Methods section).

n/a Confirmed

$\square \bigotimes$ The exact sample size $(n)$ for each experimental group/condition, given as a discrete number and unit of measurement

$\square \bigotimes$ An indication of whether measurements were taken from distinct samples or whether the same sample was measured repeatedly

$\square$ The statistical test(s) used AND whether they are one- or two-sided

Only common tests should be described solely by name; describe more complex techniques in the Methods section.

Х $\square$ A description of all covariates tested

Х $\square$ A description of any assumptions or corrections, such as tests of normality and adjustment for multiple comparisons

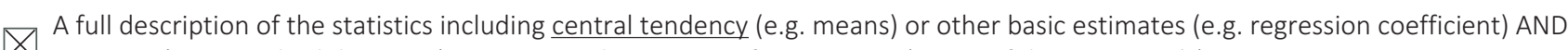

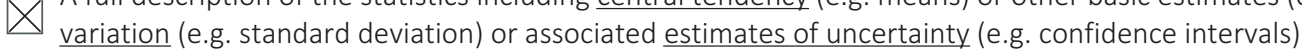

$\varnothing$ For null hypothesis testing, the test statistic (e.g. $F, t, r$ ) with confidence intervals, effect sizes, degrees of freedom and $P$ value noted

Give P values as exact values whenever suitable.

Х $\square$ For Bayesian analysis, information on the choice of priors and Markov chain Monte Carlo settings

Х $\square$ For hierarchical and complex designs, identification of the appropriate level for tests and full reporting of outcomes

Х $\square$ Estimates of effect sizes (e.g. Cohen's $d$, Pearson's $r$ ), indicating how they were calculated

$\varnothing$ Clearly defined error bars

State explicitly what error bars represent (e.g. SD, SE, CI)

Our web collection on statistics for biologists may be useful.

\section{Software and code}

Policy information about availability of computer code

Data collection BD FACS Diva software v8.0.1, SoftMax Pro 5.4.1, ZEN Lite 2012, QuantStudio Real-time PCR Software v1.3, UNICORN 5.01, NIS-Elements F 4.00.00, Immunospot 5.1

Data analysis

GraphPad Prism6 was used for data analysis and plotting.

FlowJo was used for flow cytometry analysis.

ZEN Lite 2012 and Fiji ImageJ were used for immunofluoresence image analysis.

For manuscripts utilizing custom algorithms or software that are central to the research but not yet described in published literature, software must be made available to editors/reviewers upon request. We strongly encourage code deposition in a community repository (e.g. GitHub). See the Nature Research guidelines for submitting code \& software for further information. 
Policy information about availability of data

All manuscripts must include a data availability statement. This statement should provide the following information, where applicable:

- Accession codes, unique identifiers, or web links for publicly available datasets

- A list of figures that have associated raw data

- A description of any restrictions on data availability

The datasets generated during and/or analysed during the current study are available from the corresponding author on reasonable request.

\section{Field-specific reporting}

Please select the best fit for your research. If you are not sure, read the appropriate sections before making your selection.

$\bigotimes$ Life sciences $\quad \square$ Behavioural \& social sciences $\square$ Ecological, evolutionary \& environmental sciences

For a reference copy of the document with all sections, see nature.com/authors/policies/Reportingsummary-flat.pdf

\section{Life sciences study design}

All studies must disclose on these points even when the disclosure is negative.

Sample size We did not predetermine sample size using software. The exact $\mathrm{n}$ values used to calculate the statistics are provided and a reasonable sample size was chosen to ensure adequate reproducibility of results.

Data exclusions No data were excluded from the analysis.

Replication All experiments were performed at least twice with similar results.

Randomization Mice were randomized into different groups.

Blinding Blinding was not performed due to the unambiguous nature of measurements and systematic analyses used in these experiments.

\section{Reporting for specific materials, systems and methods}

Materials \& experimental systems

\begin{tabular}{l|l}
\hline n/a Involved in the study \\
$\square$ Unique biological materials \\
$\square$ Antibodies \\
$\square$ Animals and other organisms
\end{tabular}

\begin{tabular}{l|l}
\multicolumn{2}{l}{ Methods } \\
\hline n/a Involved in the study \\
$\square$ ChIP-seq \\
$\square$ MRI-based neuroimaging
\end{tabular}

\section{Antibodies}

Antibodies used 
Streptavidin APC/Cy7 (Cat. 405208; Lot: B215107; Biolegend)

I-b. For Tfh cells staining (Fig 5a)

Thy1.1 PerCP/Cy5.5 (Clone: OX-7; Cat. 202516; Lot: B178918; Biolegend)

TCR V $\alpha 2$ PE (Clone: B20.1; Cat. 12-5812-82; Lot: E01800-1634; eBioscience)

CD4 PE/Cy7 (Clone: GK1.5; Cat. 100422; Lot: 1936945; Biolegend)

PD1 APC (Clone: J43; Cat. 17-9985-82; Lot:4335542; eBioscience)

CXCR5 biotin (Clone: SPRCL5; Cat. 13-7185-82; Lot: 4337044; eBioscience)

Streptavidin APC/Cy7 (Cat. 405208; Lot: B215107; Biolegend)

I-c. For GC B cells staining (Fig 5b)

B220 PE (Clone: RA3-6B2; Cat. 12-0452; Lot: E01249-1634; eBioscience)

GL7 Alexa Fluor488 (Clone: GL-7; Cat. 53-5902-82; Lot: E11670-1635; eBioscience)

FAS PE/Cy7 (Clone: Jo2; Cat. 557653; Lot: 7018663; BD Pharmingen ${ }^{\mathrm{TM}}$ )

I-d. For T cell activation (Fig 5d, 5e)

CD69 PE (Clone: H1.2F3; Cat. 12-0691; Lot: E01333-1634; eBioscience)

CD25 FITC (Clone: PC61; Cat. 102006; Lot: B126955; Biolegend)

CD44 APC/Cy7 (Clone: IM7; Cat. 103028; Lot:B262797; Biolegend)

CD62L AF700 (Clone: MEL-14; Cat. 56-0621-82; Lot: 4306534; eBioscience)

I-e. For B cell activation (Fig 6a,6g)

B220 APC (Clone: RA3-6B2; Cat. 103212; Lot: B208579; Biolegend)

CD86 PE (Clone: GL1; Cat. 12-0862-81; Lot: 4294065; eBioscience)

CD69 PE (Clone: H1.2F3; Cat. 12-0691-82; Lot: E01333-1634; eBioscience)

CD45.1 FITC (Clone: A20; Cat. 110705; Lot: B202563; Biolegend)

CD45.2 PerCP/Cy5.5 (Clone: 104; Cat. 45-0454-82; Lot: 4336370; eBioscience)

I-f. For human macrophage analysis (Fig S7)

HLA-DR APC/Cy7 (Clone: L243; Cat. 307617; Lot: B272894; Biolegend)

CD14 PE (Clone: M5E2; Cat. 301806; Lot: B246225; Biolegend)

CD209 APC (Clone: 9E9A8; Cat. 330107; Lot: B279429; Biolegend)

Human TruStain FcXTM (Cat. 422302; Lot: B291618; Biolegend)

The working concentration of all the antibodies for FACS is $2.5 \mu \mathrm{g} / \mathrm{ml}$.

II. For immunofluorescence assay (Fig 4a, Fig 4e, Fig 5c, Fig 6e, 6f Fig S3b, Fig S10a) Full length GFP polyclonal antibody (Cat. 632592; Lot: 1510024; Clontech; 1:1000,) SIGNR1 APC (Clone: 22D1; Cat. 17-2093-80; Lot: 4326927; eBioscience)

CD169 PE (Clone: 3D6.112; Cat. 142403; Lot: B250778; Biolegend)

CD11c FITC (Clone: N418; Cat. 117035; Lot: B244373; Biolegend)

B220 PE (Clone: RA3-6B2; Cat. 12-0452-82; Lot: E01249-1634; eBioscience)

B220 eFluor 450 (Clone: RA3-6B2; Ref: 48-0452-80; Lot: 4339476; Invitrogen)

GL7 Alexa Fluor488 (Clone: GL-7; Cat. 53-5902-82; Lot: E11670-1635; eBioscience)

CD45.1 efluor 450 (Clone: A20; Cat. 48-0453; Lot: E08504-1632; eBioscience)

CD45.2 Biotin (Clone: 104; Cat. 13-0454; Lot: E02536-1631; eBioscience)

Streptavidin FITC (Cat. 11-4317-87; Lot: 4323304; eBioscience)

The working concentration of all the antibodies for immunofluorescence is $2.5 \mu \mathrm{g} / \mathrm{ml}$.

III. For immunological histological chemistry (IHC) (Fig 3f, Fig S2C) (1:900 dilution)

Polyclonal rabbit anti-Hepatitis B virus core antigen (HBcAg) (Cat. B0586; Lot: 10048815; Dako)

IV. For ELISA (1:5000 dilution)

Horseradish peroxidase (HRP) conjugated goat anti-mouse IgG (H+L) (Cat. ZB2305; Lot: 133499; ZSGB-BIO)

Peroxidase-conjugated Goat anti-mouse IgG subclass I (Cat. SA00012-1; ProteinTech)

Peroxidase-conjugated Goat anti-mouse IgG2c (Cat. SA00012-4, ProteinTech)

Peroxidase-conjugated Goat anti-mouse IgM (Cat. SA00012-6; ProteinTech)

\section{For ELISPOT}

Purified anti-mouse IFN- $\gamma$ (Cat. 51-2525KC; Lot: 5295506; BD Biosciences) (1:200 dilution)

Biotinylated anti-mouse IFN-Y (Cat. 51-1818KZ; Lot: 6125718; BD Biosciences) (1:250 dilution)

Streptavidin-HRP (Cat. 51-9000209; Lot: 6225988; BD Biosciences) (1:100 dilution)

Donkey Anti-Mouse IgG, Biotin Conjugated (Cat:CW0225; Lot:1915F; CWbio) (1:1000 dilution)

VI. For IFNy blocking

InVivoMAb anti-mouse IFNץ (Cat\#BE0055; Lot: 699718S1; BioXCell)

All the antibodies used are from commercial sources and have been validated by the vendors. Validation data are available on the manufacturer's website.

CD19 AF700 (Clone: 6D5; Cat. 115528; Lot: 205B249811; Biolegend)

https://www.biolegend.com/en-us/products/alexa-fluor-700-anti-mouse-cd19-antibody-3391

B220 PE/Cy7 (Clone: RA3-6B2; Cat. 25-0452-82; Lot: E07569-1636; eBioscience)

https://www.thermofisher.com/cn/zh/antibody/product/CD45R-B220-Antibody-clone-RA3-6B2-Monoclonal/25-0452-81

CD11C PE (Clone: N418; Cat. 117308; Lot: B256109; Biolegend)

https://www.biolegend.com/en-us/products/pe-anti-mouse-cd11c-antibody-1816

CD11c FITC (Clone: N418; Cat. 117305; Lot: B244373; Biolegend)

https://www.biolegend.com/en-us/products/fitc-anti-mouse-cd11c-antibody-1815

I-A/I-E APC/CY7 (Clone: M5/114.15.2; Cat. 107628; Lot: B248055; Biolegend)

https://www.biolegend.com/en-us/products/apccyanine7-anti-mouse-i-a-i-e-antibody-5966 
CD11b PerCP/Cy5.5 (Clone: M1/70; Cat. 45-0112-82; Lot: 1929457; eBioscience)

https://www.thermofisher.com/antibody/primary/query/45-0112

SIGNR1 APC (Clone: 22D1; Cat. 17-2093-80; Lot: 4326927; eBioscience)

https://www.thermofisher.com/cn/zh/antibody/product/CD209b-SIGN-R1-Antibody-clone-eBio22D1-22D1-

Monoclonal/17-2093-80

CD169 PE (Clone: 3D6.112; Cat. 142403; Lot: B250778; Biolegend)

https://www.biolegend.com/en-us/products/pe-anti-mouse-cd169-siglec-1-antibody-7522

F4/80 PE/Cy7 (Clone: BM8; Cat. 123113; Lot: B237342; Biolegend)

https://www.biolegend.com/en-us/products/pe-cy7-anti-mouse-f4-80-antibody-4070

CD103 FITC (Clone: 2E 7; Cat. 11-1031-82; Lot: 4301319; eBioscience)

https://www.thermofisher.com/antibody/primary/query/11-1031

CXCR5 biotin (Clone: SPRCL5; Cat. 13-7185-82; Lot: 4337044; eBioscience)

https://www.thermofisher.com/cn/zh/antibody/product/CD185-CXCR5-Antibody-clone-SPRCL5-Monoclonal/13-7185-82

Streptavidin APC/Cy7 (Cat. 405208; Lot: B215107; Biolegend)

https://www.biolegend.com/en-us/products/apc-cy7-streptavidin-1471

Thy1.1 PerCP/Cy5.5 (Clone: OX-7; Cat. 202516; Lot: B178918; Biolegend)

https://www.biolegend.com/en-us/products/percpcyanine55-anti-rat-cd90mouse-cd901-thy-11-antibody-4514

TCR V $\alpha 2$ PE (Clone: B20.1; Cat. 12-5812-82; Lot: E01800-1634; eBioscience)

https://www.thermofisher.com/antibody/primary/query/12-5812

CD4 PE/Cy7 (Clone: GK1.5; Cat. 100422; Lot: 1936945; Biolegend)

https://www.biolegend.com/en-us/products/pe-cy7-anti-mouse-cd4-antibody-1919

PD1 APC (Clone: J43; Cat. 17-9985-82; Lot:4335542; eBioscience)

https://www.thermofisher.com/cn/zh/antibody/product/CD279-PD-1-Antibody-clone-J43-Monoclonal/17-9985-82

B220 PE (Clone: RA3-6B2; Cat. 12-0452-82; Lot: E01249-1634; eBioscience)

https://www.thermofisher.com/antibody/primary/query/12-0452

GL7 Alexa Fluor488 (Clone: GL-7; Cat. 53-5902-82; Lot: E11670-1635; eBioscience)

https://www.thermofisher.com/cn/zh/antibody/product/GL7-Antibody-clone-GL-7-GL7-Monoclonal/53-5902-82

FAS PE/Cy7 (Clone: Jo2; Cat. 557653; Lot: 7018663; BD Pharmingen ${ }^{\text {TM }}$ )

https://www.fishersci.com/shop/products/anti-cd95-pe-cy-7-clone-jo2-bd/bdb557653\#?keyword=557653

CD69 PE (Clone: H1.2F3; Cat. 12-0691-82; Lot: E01333-1634; eBioscience)

https://www.thermofisher.com/antibody/primary/query/12-0691

CD25 FITC (Clone: PC61; Cat. 102006; Lot: B126955; Biolegend)

https://www.biolegend.com/en-us/products/fitc-anti-mouse-cd25-antibody-422

CD44 APC/Cy7 (Clone: IM7; Cat. 103028; Lot:B262797; Biolegend)

https://www.biolegend.com/en-us/products/apc-cy7-anti-mouse-human-cd44-antibody-3933

CD62L AF700 (Clone: MEL-14; Cat. 56-0621-82; Lot: 4306534; eBioscience)

https://www.thermofisher.com/antibody/primary/query/CD62L\%20AF700

B220 APC (Clone: RA3-6B2; Cat. 103212; Lot: B208579; Biolegend)

https://www.biolegend.com/en-us/products/apc-anti-mouse-human-cd45r-b220-antibody-442

CD86 PE (Clone: GL1; Cat. 12-0862-81; Lot: 4294065; eBioscience)

https://www.thermofisher.com/cn/zh/antibody/product/CD86-B7-2-Antibody-clone-GL1-Monoclonal/12-0862-81

CD45.1 FITC (Clone: A20; Cat. 110705; Lot: B202563; Biolegend)

https://www.biolegend.com/en-us/products/fitc-anti-mouse-cd45-1-antibody-198

CD45.2 PerCP/Cy5.5 (Clone: 104; Cat. 45-0454-82; Lot: 4336370; eBioscience)

https://www.thermofisher.com/antibody/primary/query/45-0454

HLA-DR APC/Cy7 (Clone: L243; Cat. 307617; Lot: B272894; Biolegend)

https://www.biolegend.com/en-us/products/apc-cyanine7-anti-human-hla-dr-antibody-2863

CD14 PE (Clone: M5E2; Cat. 301806; Lot: B246225; Biolegend)

https://www.biolegend.com/en-us/products/pe-anti-human-cd14-antibody-796

CD209 APC (Clone: 9E9A8; Cat. 330107; Lot: B279429; Biolegend)

https://www.biolegend.com/en-us/products/apc-anti-human-cd209-dc-sign-antibody-4886

Human TruStain FcXTM (Cat. 422302; Lot: B291618; Biolegend)

https://www.biolegend.com/en-us/products/human-trustain-fcx-fc-receptor-blocking-solution-6462

Full length GFP polyclonal antibody (Cat. 632592; Lot: 1510024; Clontech; 1:1000,)

https://www.takarabio.com/products/antibodies-and-elisa/fluorescent-protein-antibodies/green-fluorescent-protein-

antibodies?catalog=632592

B220 eFluor 450 (Clone: RA3-6B2; Ref: 48-0452-80; Lot: 4339476; Invitrogen)

https://www.thermofisher.com/cn/zh/antibody/product/CD45R-B220-Antibody-clone-RA3-6B2-Monoclonal/48-0452-80

CD45.1 efluor 450 (Clone: A20; Cat. 48-0453-82; Lot: E08504-1632; eBioscience)

https://www.thermofisher.com/antibody/primary/query/48-0453

CD45.2 Biotin (Clone: 104; Cat. 13-0454-82; Lot: E02536-1631; eBioscience)

https://www.thermofisher.com/antibody/primary/query/13-0454

Streptavidin FITC (Cat. 11-4317; Lot: 4323304; eBioscience)

https://www.thermofisher.com/order/catalog/product/11-4317-87?SID=srch-hj-11-4317-87

Horseradish peroxidase (HRP) conjugated goat anti-mouse IgG (H+L) (Cat. ZB2305; Lot: 133499; ZSGB-BIO)

http://www.zsbio.com/product/ZB-2305

Peroxidase-conjugated Goat anti-mouse IgG subclass I (Cat. SA00012-1; ProteinTech)

http://www.ptgcn.com/Products/HRP-conjugated-Affinipure-Goat-Anti-Mouse-IgG,Fc\%CE\%B3Subclass-1-Specific-secondary-

antibody.htm

Peroxidase-conjugated Goat anti-mouse IgG2c (Cat. SA00012-4, ProteinTech)

https://www.ptgcn.com/Products/HRP-conjugated-Affinipure-Goat-Anti-Mouse-IgG,-Fc\%ce\%b3Subclass-2c-Specific-secondaryantibody.htm

Peroxidase-conjugated Goat anti-mouse IgM (Cat. SA00012-6; ProteinTech)

https://www.ptgcn.com/Products/HRP-conjugated-Affinipure-Goat-Anti-Mouse-IgM,-\%ce\%bc-Chain-Specific-secondary-

antibody.htm 
Purified anti-mouse IFN- $\gamma$ (Cat. 51-2525KC; Lot: 5295506; BD Biosciences)

Biotinylated anti-mouse IFN- $\gamma$ (Cat. 51-1818KZ; Lot: 6125718; BD Biosciences)

Streptavidin-HRP (Cat. 51-9000209; Lot: 6225988; BD Biosciences)

https://www.bdbiosciences.com/us/applications/research/t-cell-immunology/th-1-cells/immunoassays/elispot/mouse/mouseifn--elispot-set/p/551083

Donkey Anti-Mouse IgG, Biotin Conjugated (Cat:CW0225; Lot:1915F; CWbio)

http://www.cwbiotech.com/product/19887.html

InVivoMAb anti-mouse IFNy (Cat\#BE0055; Lot: 699718S1; BioXCell)

https://bxcell.com/product/invivomab-anti-m-ifngamma/

\section{Eukaryotic cell lines}

Policy information about cell lines

Cell line source(s)

HepG2-hNTCP cell line was generated by Dr. Wenhui Li (National Institute of Biological Sciences, Beijing, China). Ref: Elife. 2012 Nov 13;3. doi: 10.7554/eLife.00049.

Authentication

No authentication was carried out. Cell morphology and behavior such as infection by HBV was consistent with expectations.

Mycoplasma contamination

The cell line was tested to be negative for Mycoplasma.

Commonly misidentified lines

(See ICLAC register)

No commonly misidentified cell lines were used in this study.

\section{Animals and other organisms}

Policy information about studies involving animals; ARRIVE guidelines recommended for reporting animal research

Laboratory animals

Wild type C57BL/6 mice and BALB/c mice were obtained from Vital River Laboratory Animal Technology Co. (Beijing, China). CD11c-DTR mice and OT-II TCR transgenic mice were purchased from The Jackson Laboratory. MD4 transgenic mice were kindly provided by Dr. Baidong Hou (Chinese Academy of Science Key Laboratory for Infection and Immunity, Institute of Biophysics, Chinese Academy of Sciences, Beijing, China). Cxcr5-/- mice were kindly provided by Dr. Hai Qi (Institute for Immunology, School of Medicine, Tsinghua University, Beijing, China). Signr1-/- mice were generated by Cyagen Biosciences Inc. (Suzhou, China). For the detection of antibody response in wild type C57BL/6 mice and BALB/c mice, 6-10 weeks-old female mice were used. In the AAV-HBV1.3 associated protective assay, 6-10 weeks-old C57BL/6N male mice were used. In the AAV-HBV1.3 associated therapeutic assay, 4-5 weeks-old C57BL/6N male mice were used. For the using of transgenic mice, age and sex matched female or male mice were used for each experiment.

Wild animals

No wild animals were involved.

Field-collected samples

No field-collected samples were used.

\section{Human research participants}

Policy information about studies involving human research participants

Population characteristics Human fresh distal mesenteric lymph nodes from neuroblastoma patients of 2.5-14 years old were derived from the patients who underwent surgery at Department of Pediatric Surgical Oncology, Children's Hospital of Chongqing Medical University, Chongqing, P.R China. Patients are HIV, HBV, HCV negative. Patients with acute infection, fever or diarrhoea were also excluded. No endocrine therapy, chemotherapy, or radiotherapy was given to patients before surgery.

Recruitment

We used available mesenteric lymph nodes samples from the patients. The patients were informed, and they signed consent forms acknowledging the use of their resected tissues for research purposes. There was no potential self-selection bias.

\section{Flow Cytometry}

Plots

Confirm that:

\The axis labels state the marker and fluorochrome used (e.g. CD4-FITC).

\The axis scales are clearly visible. Include numbers along axes only for bottom left plot of group (a 'group' is an analysis of identical markers).

$\bigotimes$ All plots are contour plots with outliers or pseudocolor plots.

$\bigotimes$ A numerical value for number of cells or percentage (with statistics) is provided.

\section{Methodology}

Sample preparation

Lymph nodes were harvested and gently minced using scissors in FACS buffer (PBS containing $2 \% \mathrm{FBS}$ ). The tissues were then digested with $0.5 \mathrm{mg} / \mathrm{ml}$ collagenase I (Sigma) and $0.04 \mathrm{mg} / \mathrm{ml}$ DNase I (Roche) at $37^{\circ} \mathrm{C}$ for $1 \mathrm{~h}$. In CXCR5 detection and ferritin- 
Instrument

Software

Cell population abundance

Gating strategy
FITC binding assay, LNs were digested with $100 \mathrm{U} / \mathrm{ml}$ CLSPA (Worthington) and $0.04 \mathrm{mg} / \mathrm{ml}$ DNase I (Roche) in 1640 medium at $37^{\circ} \mathrm{C}$ for $1 \mathrm{~h}$. The suspension was washed with cold FACS buffer, disaggregated by passing through a $70 \mu \mathrm{m}$ cell strainer (Biologix Group) and centrifuged at $500 \mathrm{~g}$ for $5 \mathrm{~min}$. Splenocytes were collected in the same manner, and red blood cells were removed with ammonium-chloride-potassium buffer.

LSRFortessa, FACS Aria III

BD FACS Diva 8.0.1 software was used for data collection and FlowJo v.10 was used for data analysis.

In mouse lymph nodes, there are $\sim 0.1 \%$ of SIGNR1+ rDC, $\sim 0.04 \%$ of SIGNR1+ miDC, $\sim 0.7 \%$ of SIGNR1+ macrophage, and $\sim 0.2 \%$ $\mathrm{F} 4 / 80+$ macrophage in single alive cell gate.

For GC B cells, $\sim 30 \%$ of B220+ cells and $0.4 \%$ of FAS+GL7+ cells in ferritin NP vaccine immunized groups and $0.09 \%$ in control. For Tfh cells, $2 \%$ of OTII+CD4+ cells and $0.1 \%$ of CXCR5+PD1+GL7+ cells in ferritin NP vaccine immunized group, 1\% of OTII + CD4+ cells and $0.006 \%$ of CXCR5+PD1+GL7+ cells in control.

In human lymph nodes, there are $\sim 0.4 \%$ of CD14+ macrophage and $\sim 0.17 \%$ of DC-SIGN+ macrophage in single alive cell gate. For flow sorting, post-sort cells were analyzed on BD Aria III and the purity was at least $95 \%$.

I. For mouse DC and macrophage analysis and sorting

I-a. For naïve LN, single alive lymphocytes were first gated with CD19 and B220, then non-B cells were gated with CD11c and $\mathrm{MHCl}, \mathrm{CD} 11 \mathrm{c}$ high cells were resident DC, MHCII high cells were migratory DC, others were non-DCs. DCs were then gated with SIGNR1 and CD11b. CD11b+ non-DCs were then gated with CD169 and F4/80 or SIGNR1 and F4/80.

I-b. For immunized LN, CD11c+MHCII+ DCs were gated with CD103, CD103- cells were resident DC, CD103+ cells were migratory DC; macrophages were gated as the same as above.

II. For human macrophage analysis

HLA-DR+ CD14+ macrophages were gated and identified into CD209+ and CD209- populations.

III. For Tfh staining and GC B cells

Tfh cells: single alive lymphocytes were gated with Thy1.1+TCR V $\alpha 2+C D 4+$, and finally gated with CXCR5+PD-1+ cells or GL7+PD-1 + cells.

GC B cells: single alive lymphocytes were gated with B220+ cells, and finally gated with FAS+GL7+ cells.

Tick this box to confirm that a figure exemplifying the gating strategy is provided in the Supplementary Information. 